\title{
Discovery and analysis of p-mode and g-mode oscillations in the A-type primary of the eccentric binary HD $209295^{\star}$
}

\author{
G. Handler, ${ }^{1} \dagger$ L. A. Balona, ${ }^{1}$ R. R. Shobbrook, ${ }^{2,3}$ C. Koen, ${ }^{1}$ A. Bruch, ${ }^{4}$ \\ E. Romero-Colmenero, ${ }^{1}$ A. A. Pamyatnykh, ${ }^{5,6}$ B. Willems, ${ }^{7}$ L. Eyer, ${ }^{8,9}$ D. J. James ${ }^{10,11,12,13}$ \\ and T. Maas ${ }^{8}$ \\ ${ }^{1}$ South African Astronomical Observatory, PO Box 9, Observatory 7935, South Africa \\ ${ }^{2}$ PO Box 518, Coonabarabran, NSW 2357, Australia \\ ${ }^{3}$ Research School of Astronomy and Astrophysics, Australian National University, Weston Creek PO, ACT 2611, Australia \\ ${ }^{4}$ Labóratorio Nacional de Astrofísica, Itajubá, Brazil \\ ${ }^{5}$ Copernicus Astronomical Center, ul. Bartycka 18, 00-716 Warsaw, Poland \\ ${ }^{6}$ Institute of Astronomy, Russian Academy of Sciences, Pyatnitskaya 48, 109017 Moscow, Russia \\ ${ }^{7}$ Department of Physics and Astronomy, The Open University, Walton Hall, Milton Keynes MK7 6AA \\ ${ }^{8}$ Instituut voor Sterrenkunde, Katholieke Universiteit Leuven, B-3001 Leuven (Heverlee), Belgium \\ ${ }^{9}$ Astrophysical Sciences Department, Princeton University, Princeton, NJ 08544, USA \\ ${ }^{10}$ Observatoire de Genève, Chemin des Maillettes 51, CH-1290 Sauverny, Switzerland \\ ${ }^{11}$ Laboratoire d'Astrophysique, Observatoire de Grenoble, Université Joseph Fourier, BP 53, F-38041, Grenoble Cedex 9, France \\ ${ }^{12}$ School of Physics \& Astronomy, University of St Andrews, North Haugh, St Andrews, Fife KY16 9SS \\ ${ }^{13} 475$ N. Charter Street, 5534 Sterling Hall, Madison, WI 53706-1582, USA
}

Accepted 2001 December 18. Received 2001 December 17; in original form 2001 October 18

\begin{abstract}
A B S T R A C T
We have discovered both intermediate-order gravity mode and low-order pressure mode pulsation in the same star, HD 209295. It is therefore both a $\gamma$ Doradus and a $\delta$ Scuti star, which makes it the first pulsating star to be a member of two classes.

The analysis of our $128 \mathrm{~h}$ of multisite spectroscopic observations carried out over two seasons reveals that the star is a single-lined spectroscopic binary with an orbital period of $3.10575 \pm 0.00010 \mathrm{~d}$ and an eccentricity of $0.352 \pm 0.011$. Only weak pulsational signals are found in both the radial velocity and line-profile variations, but we have succeeded in showing that the two highest-amplitude $\gamma$ Doradus pulsation modes are consistent with $\ell=1$ and $|m|=1$.
\end{abstract}

These two modes dominated our $280 \mathrm{~h}$ of $B V I_{\mathrm{C}}$ multisite photometry, also obtained over two seasons. We detected altogether ten frequencies in the light variations, one in the $\delta$ Scuti regime and nine in the $\gamma$ Doradus domain. Five of the $\gamma$ Doradus frequencies are exact integer multiples of the orbital frequency. This observation leads us to suspect they are tidally excited. Attempts to identify modes from the multicolour photometry failed.

We performed model calculations and a stability analysis of the pulsations. The frequency range in which $\delta$ Scuti modes are excited agrees well with observations. However, our models do not show excitation of $\gamma$ Doradus pulsations, although the damping is smaller in the observed range. We also investigated tidal excitation of $\gamma$ Doradus modes. Some of the observed harmonics of the orbital period were found to be unstable. The observed orbital harmonics which are stable in the models can be understood as linear combinations of the unstable modes.

We could not detect the secondary component of the system in infrared photometry, suggesting that it may not be a main-sequence star. Archival data of this star show that it has a strong ultraviolet (UV) excess, the origin of which is not known. The orbit of the primary is consistent with a secondary mass of $M>1.04 \mathrm{M}_{\odot}$, which is indicative of a neutron star, although a white dwarf companion is not ruled out.

\footnotetext{
^ Dedicated to the memory of A. W. J. Cousins, discoverer of the variability

of $\gamma$ Doradus.

$\dagger$ E-mail: gerald@saao.ac.za
} 
Key words: binaries: close - binaries: spectroscopic - stars: individual: HD 209295 - stars: neutron - stars: oscillations $-\delta$ Scuti.

\section{INTRODUCTION}

Four different classes of multimode pulsating variables are found near the intersection of the classical instability strip and the main sequence. However, asteroseismology (probing the stellar interior through such pulsations) of these stars has proven to be more difficult than expected. None the less, the example of helioseismology and its enormous reward in terms of the physical understanding of the Sun's interior (e.g. see Gough 2000) has been a great motivation for continuing efforts in probing the structure of these stars by means of their pulsational properties.

Many attempts to detect solar-type oscillations in stars have resulted in inconclusive results, although success has been claimed in some instances (e.g. Kjeldsen et al. 1995). Because of their extremely low amplitude, it is only recently (e.g. Bedding et al. 2002) that we are beginning to see detections which may be considered significant. Successful asteroseismology based on the analysis of solar-type oscillations now appears immanent.

The rapidly oscillating Ap stars (see Kurtz \& Martinez 2000 for a recent comprehensive review) are high-radial order p-mode pulsators. Their pulsation spectra resemble that of the Sun. In these stars, only a few independent modes are observed. Moreover, the presence of a strong magnetic field is expected to modify the frequencies and eigenfunctions of the modes. Successful modelling of these pulsations does not seem possible at this time. Nevertheless, some progress is currently being made (e.g. Cunha \& Gough 2000).

The third group of pulsators in this region of the HertzsprungRussell (HR) diagram are the $\delta$ Scuti ( $\delta$ Sct) stars, which are lowradial order p- and probably mixed-mode pulsators (see Breger $\&$ Montgomery 2000). For some of these stars, tens of pulsations modes have been detected and their frequencies determined with high precision (see e.g. Handler et al. 2000). Although mean densities, and even asteroseismological distances (Handler et al. 1997) have been estimated, the problem of mode identification and shortcomings in the stellar models are major difficulties (see e.g. Pamyatnykh et al. 1998 for a discussion).

The $\gamma$ Doradus ( $\gamma$ Dor) stars are high-overtone gravity (g) mode pulsators (Kaye et al. 1999a). In these stars only a few modes are excited to observable amplitude amongst a dense forest of possible modes. As a result, accurate starting values of the basic stellar parameters are required to enable observed and calculated frequencies to be matched.

In principle, it is possible for $\delta$ Sct and $\gamma$ Dor pulsations to coexist in a star as they occupy overlapping regions in the HR diagram. This offers new opportunities for successful asteroseismology, as noted by Handler (1999a). In such stars it may be possible to use the $\delta$ Sct pulsations to place strong constraints on the stellar parameters, easing the mode identification problem of the $\gamma$ Dor pulsations. In this way it may be possible to obtain information about the deep interior which is unobtainable for pure $\delta$ Sct stars.

Consequently, Handler \& Shobbrook (2002) searched for $\delta$ Sct pulsations in all known candidate $\gamma$ Dor stars located within the $\delta$ Sct instability strip and accessible from intermediate southern geographical latitudes. About one third of all non-Am and non-Ap stars in the lower instability strip are indeed $\delta$ Sct stars (Breger 1975). Because Am and Ap stars are rare (if not absent) amongst the $\gamma$ Dor stars (Handler 1999a), detection of some 'hybrid' stars may be expected if $\delta$ Sct and $\gamma$ Dor pulsations are not mutually exclusive. In this paper we report the discovery and analysis of such a star, HD 209295 (Sangam Mani).

This is a $V=7.3 \mathrm{mag}$ southern $\left(\delta=-64^{\circ}\right)$ star of spectral type A9/F0 V (Houk \& Cowley 1975). It was discovered as variable by the Hipparcos mission (ESA 1997). Handler (1999a) performed a frequency analysis of these data and detected four periods; we show the results in Fig. 1 and Table 1. We are aware that a search for multiple periods in Hipparcos photometry is difficult and can lead to spurious results (Eyer \& Grenon 1998). However, the case of HD 209295 is so simple and convincing that an error in the analysis is considered improbable. The multiperiodicity leaves no doubt that HD 209295 is a bona fide $\gamma$ Dor star.

HD 209295 is considerably hotter than all other $\gamma$ Dor stars. Handler (1999a) suggested that the published Strömgren $(b-y)$ of this star (Twarog 1980) is too blue compared to $(B-V)$. Handler (1999b) obtained new Strömgren photometry and confirmed the

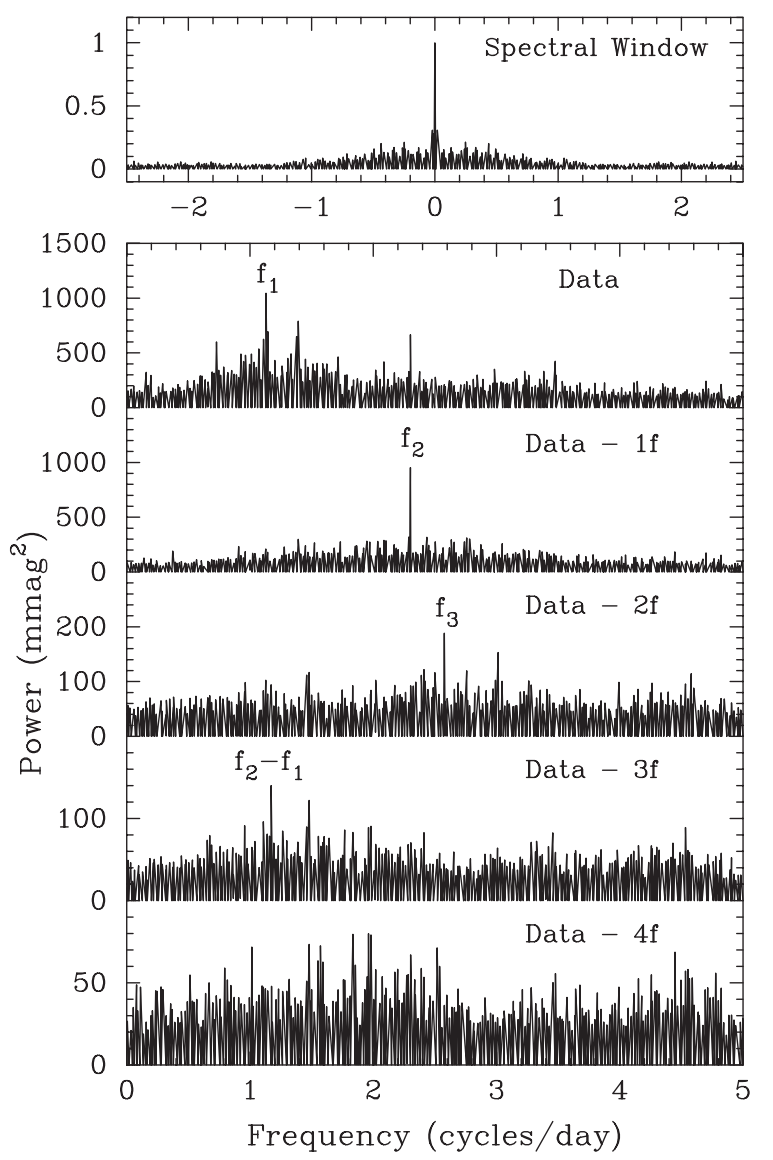

Figure 1. Spectral window and amplitude spectra of Hipparcos photometry of HD 209295 with consecutive pre-whitening of detected frequencies. The combination peak $f_{2}-f_{1}$ is only considered significant because it occurs at a 'predicted' frequency. 
Table 1. Multifrequency solution for the $\gamma$ Dor variability of HD 209295 from Hipparcos observations. Formal error estimates were derived from Montgomery \& O'Donoghue (1999). The signal-to-noise ratio (S/N) was calculated following Breger et al. (1993); a $\mathrm{S} / \mathrm{N} \geq 4$ corresponds to a significant detection.

\begin{tabular}{lccr}
\hline ID & $\begin{array}{c}\text { Frequency } \\
\left(\text { cycle d }^{-1}\right)\end{array}$ & $\begin{array}{c}H_{\mathrm{p}} \text { Amplitude } \\
(\mathrm{mmag})\end{array}$ & $\mathrm{S} / \mathrm{N}$ \\
\hline$f_{1}$ & $1.12957 \pm 0.00002$ & $37 \pm 2$ & 12 \\
$f_{2}$ & $2.30222 \pm 0.00003$ & $31 \pm 2$ & 10 \\
$f_{3}$ & $2.57579 \pm 0.00005$ & $16 \pm 2$ & 5 \\
$f_{4}=f_{2}-f_{1}$ & $1.1726 \pm 0.0001$ & $12 \pm 2$ & 4 \\
\hline
\end{tabular}

Table 2. Published uvby $\beta$ photometry of HD 209295. The differences in the magnitudes and photometric indices are consistent with stellar pulsation. When brighter the star it is bluer and the $c_{1}$ and $\mathrm{H}_{\beta}$ indices are larger.

\begin{tabular}{cccccc}
\hline $\mathrm{V}$ & $b-y$ & $m_{1}$ & $c_{1}$ & $\beta$ & Reference \\
\hline 7.32 & 0.149 & 0.182 & 0.819 & 2.781 & Twarog (1980) \\
7.29 & 0.139 & 0.185 & 0.840 & 2.821 & Handler (1999b)
\end{tabular}

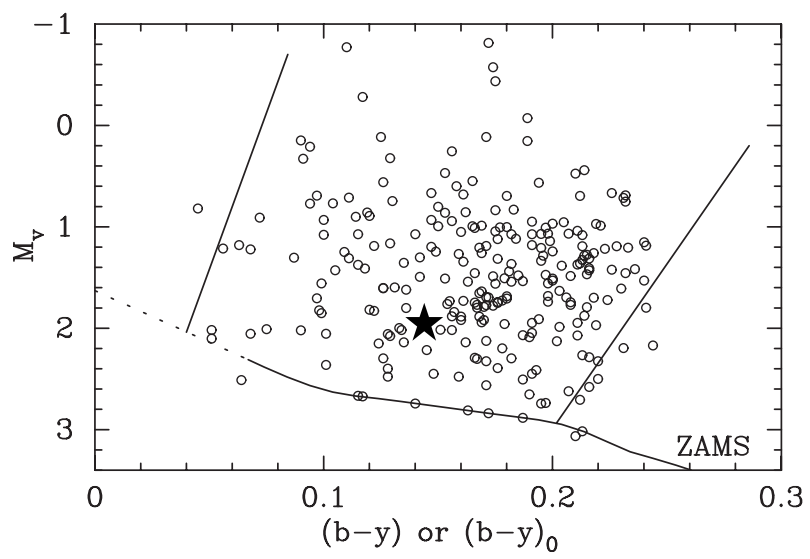

Figure 2. The location of HD 209295 (star symbol) in the colourmagnitude diagram compared to 252 known $\delta$ Sct stars (open circles) with available Strömgren photometry from the catalogue of Rodriguez, LópezGonzález \& López de Coca (2000). The blue and red edges of the $\delta$ Sct instability strip (Rodriguez \& Breger 2001) are indicated.

discrepancy (cf. Table 2). Grenier et al. (1999) made two measurements of the radial velocity of the star using an echelle spectrograph and the cross-correlation technique. They did not comment on binarity or any other peculiarity.

Using the mean of the Strömgren indices from Table 2 (which indicate zero reddening), the effective temperature and gravity of HD 209295 can be estimated. Kurucz's (1991) calibration yields $T_{\text {eff }}=7750 \pm 100 \mathrm{~K}, \log g=4.10 \pm 0.05$.

The Hipparcos parallax of HD 209295 is $8.19 \pm 0.72$ mas. Using $V_{0}=7.32 \pm 0.01, B C=-0.01 \pm 0.01$, the parallax gives $\log L / \mathrm{L}_{\odot}=1.15 \pm 0.08$. With $\log T_{\text {eff }}=3.889$ as determined above, $R / \mathrm{R}_{\odot}=2.08 \pm 0.09$ and, by means of evolutionary tracks calculated using the Warsaw-New Jersey code (see e.g. Pamyatnykh et al. 1998), a mass of $1.84 \pm 0.07 \mathrm{M}_{\odot}$ is determined. We can then also calculate a semi-independent second value for $\log g$, namely $4.07 \pm 0.05$, consistent with the previous value. HD 209295 is therefore the hottest $\gamma$ Dor star known to date; it lies in the middle of the $\delta$ Sct instability strip on the main sequence (Fig. 2).

Because of its location in the colour-magnitude diagram, HD 209295 is a good candidate $\delta$ Sct star. Indeed, multiperiodic $\delta$ Sct pulsations were found by Handler \& Shobbrook (2002), thus placing HD 209295 as a member of two classes of pulsating star. Spectroscopic and photometric follow-up observations were organized immediately after this discovery, and a multisite campaign was conducted in 2000 August. We report the results of these campaigns.

\section{OBSERVATIONS}

\subsection{Optical photometry}

As comparison stars, we used HD 207802 (B9/B9.5V, $V=8.0$ ) and HD 209829 (F3V, $V=7.7$ ) in our photoelectric photometry. Measurements were obtained at the 1.0-, 0.75- and $0.5-\mathrm{m}$ telescopes of the Sutherland station of the South African Astronomical Observatory (SAAO), the 0.6-m telescope at Siding Spring Observatory (SSO) in Australia (Shobbrook 2000), and the 0.6-m Zeiss telescope at the Osservatório do Pico dos Dias (LNA, Brazil). Observations were conducted from 1999 October to 2000 October. Most measurements were obtained in 2000 August and September in a coordinated multisite effort. A journal of the observations is given in Table 3 .

We used the Johnson $B$ and $V$ filters as well as the Cousins $I_{\mathrm{C}}$ filter (the latter only in the year 2000) with a total integration time of $\sim 1 \mathrm{~min}$ in each filter as a compromise between good time resolution and maximum colour information. Apertures of $30-45 \operatorname{arcsec}$ on the sky were used. Sky measurements were taken at suitable intervals depending on the brightness and proximity of the Moon.

The observing sequence was chosen to result in both good coverage for the short-period $\delta$ Sct pulsations and best long-term stability. We adopted the sequence C1-V-C2-V-C1... (C1 and C2 are the comparison stars and the $\mathrm{V}$ is the variable), which resulted in one variable star measurement every $\sim 6 \mathrm{~min}$. Supplementary observations of $U B V(R I)_{\mathrm{C}}$ standard stars were also acquired.

Data reduction was performed in the standard way, i.e. corrections for coincidence losses, sky background and extinction were followed by calculating differential magnitudes between the comparison stars. The latter were examined for variability. It seems that one of the two stars is slightly variable on a time scale of about 1.1 or $10 \mathrm{~d}$ with a $V$ amplitude of 2 mmag. We investigated whether this could be due to differential colour extinction and found no support for such an interpretation. As our programme star exhibits long-term variability with much higher amplitude, we cannot determine unambiguously which of the two stars is the potential variable, but we suspect HD 209829.

In any case, we proceeded by constructing a differential target star light curve relative to the measurements of both comparison stars. We standardized these magnitude differences by using the transformation equation slopes calculated from the standard star observations mentioned above. We note that the photometric zeropoints of the different telescope/instrument combinations agreed to better than 2 mmag for each filter or colour used during the multisite campaign, but we experienced some difficulties with homogenizing the data from the discovery season. Finally, the time-base of our observations was converted to Heliocentric Julian Date (HJD). Most of the light curves obtained during the central part of the multisite campaign are shown in Fig. 3. 
Table 3. Journal of the photometric observations. The measurements before HJD 2451600 were made in the Johnson $B V$ system, whereas later observations utilized JohnsonCousins $B V I_{\mathrm{C}}$ except for one night of $B V$ observations, marked with an asterisk.

\begin{tabular}{|c|c|c|c|}
\hline Telescope & $\begin{array}{c}\text { Run start } \\
\text { HJD }-2450000\end{array}$ & $\begin{array}{l}\text { Run length } \\
\text { (h) }\end{array}$ & Observer \\
\hline SAAO $0.75-\mathrm{m}$ & 1464.335 & 3.31 & GH \\
\hline SAAO $0.75-\mathrm{m}$ & 1465.249 & 6.34 & GH \\
\hline SSO 0.6-m & 1467.033 & 2.78 & RRS \\
\hline SSO $0.6-\mathrm{m}$ & 1468.916 & 6.05 & RRS \\
\hline SSO 0.6-m & 1491.937 & 2.35 & RRS \\
\hline SSO $0.6-\mathrm{m}$ & 1498.918 & 2.35 & RRS \\
\hline SAAO $0.5-\mathrm{m}$ & 1502.282 & 1.85 & $\mathrm{GH}$ \\
\hline SAAO $0.5-\mathrm{m}$ & 1503.268 & 2.62 & GH \\
\hline SAAO $0.5-\mathrm{m}$ & 1505.267 & 2.57 & $\mathrm{GH}$ \\
\hline SAAO $0.5-\mathrm{m}$ & 1507.310 & 1.08 & $\mathrm{GH}$ \\
\hline SAAO $0.5-\mathrm{m}$ & 1509.295 & 1.63 & GH \\
\hline SSO 0.6-m & 1510.924 & 1.85 & RRS \\
\hline SAAO $0.5-\mathrm{m}$ & 1511.264 & 2.40 & $\mathrm{GH}$ \\
\hline SSO 0.6-m & 1512.933 & 2.18 & RRS \\
\hline SSO $0.6-\mathrm{m}$ & 1513.926 & 0.94 & RRS \\
\hline SSO $0.6-\mathrm{m}$ & 1516.930 & 2.04 & RRS \\
\hline SSO 0.6-m & 1517.921 & 1.20 & RRS \\
\hline SAAO $0.5-\mathrm{m}$ & 1518.266 & 0.86 & LE \\
\hline SAAO $0.5-\mathrm{m}$ & 1519.283 & 0.48 & LE \\
\hline SSO 0.6-m & 1524.930 & 1.63 & RRS \\
\hline SSO $0.6-\mathrm{m}$ & 1754.110 & 3.41 & RRS \\
\hline SSO 0.6-m & 1754.962 & 5.45 & RRS \\
\hline SAAO $0.75-\mathrm{m}$ & 1758.343 & 3.46 & ERC \\
\hline SSO 0.6-m & 1759.947 & 8.11 & RRS \\
\hline SAAO $0.75-\mathrm{m}$ & 1761.343 & 7.87 & ERC \\
\hline SAAO $0.75-\mathrm{m}$ & 1762.364 & 7.25 & ERC \\
\hline SSO 0.6-m & 1762.944 & 7.75 & RRS \\
\hline SAAO $0.75-\mathrm{m}$ & 1763.337 & 8.11 & ERC \\
\hline SAAO $0.75-\mathrm{m}$ & 1764.278 & 6.53 & ERC \\
\hline SSO 0.6-m & 1767.227 & 2.28 & RRS \\
\hline SAAO $0.75-\mathrm{m}$ & 1768.241 & 10.25 & $\mathrm{GH}$ \\
\hline SAAO $0.75-\mathrm{m}$ & 1769.239 & 10.27 & GH \\
\hline LNA $0.6-\mathrm{m}$ & 1769.819 & 0.24 & $\mathrm{AB}$ \\
\hline SAAO $0.75-\mathrm{m}$ & 1770.234 & 10.37 & $\mathrm{GH}$ \\
\hline LNA 0.6-m & 1770.514 & 6.84 & $\mathrm{AB}$ \\
\hline SAAO $0.75-\mathrm{m}$ & 1771.237 & 10.27 & $\mathrm{GH}$ \\
\hline LNA 0.6-m & 1771.498 & 3.19 & $\mathrm{AB}$ \\
\hline SAAO $0.75-\mathrm{m}$ & 1772.238 & 10.27 & GH \\
\hline SAAO $0.75-\mathrm{m}$ & 1773.226 & 10.49 & GH \\
\hline SAAO $0.75-\mathrm{m}$ & 1775.223 & 4.99 & $\mathrm{GH}$ \\
\hline LNA 0.6-m & 1775.575 & 5.59 & $\mathrm{AB}$ \\
\hline LNA 0.6-m & 1776.651 & 3.91 & $\mathrm{AB}$ \\
\hline LNA $0.6-\mathrm{m}$ & 1777.486 & 7.56 & $\mathrm{AB}$ \\
\hline SSO 0.6-m & 1777.904 & 6.14 & RRS \\
\hline SAAO $0.75-\mathrm{m}$ & 1778.493 & 3.67 & $\mathrm{GH}$ \\
\hline SAAO $1.0-\mathrm{m}^{*}$ & 1785.256 & 9.94 & $\mathrm{GH}$ \\
\hline SSO 0.6-m & 1785.918 & 8.45 & RRS \\
\hline SSO 0.6-m & 1786.991 & 0.67 & RRS \\
\hline SSO 0.6-m & 1787.898 & 9.58 & RRS \\
\hline SAAO $0.5-\mathrm{m}$ & 1789.242 & 8.74 & $\mathrm{GH}$ \\
\hline SSO 0.6-m & 1789.935 & 8.52 & RRS \\
\hline SAAO $0.5-\mathrm{m}$ & 1791.337 & 1.27 & $\mathrm{GH}$ \\
\hline SAAO 0.5-m & 1792.253 & 8.42 & $\mathrm{GH}$ \\
\hline SAAO $0.5-\mathrm{m}$ & 1850.284 & 1.51 & DJJ \\
\hline SAAO $0.5-\mathrm{m}$ & 1852.277 & 1.78 & DJJ \\
\hline SAAO $0.5-\mathrm{m}$ & 1853.332 & 0.41 & DJJ \\
\hline SAAO $0.5-\mathrm{m}$ & 1857.270 & 2.45 & DJJ \\
\hline SAAO $0.5-\mathrm{m}$ & 1858.276 & 1.82 & DJJ \\
\hline SAAO $0.5-\mathrm{m}$ & 1859.274 & 1.92 & DJJ \\
\hline SAAO $0.5-\mathrm{m}$ & 1862.278 & 2.04 & DJJ \\
\hline Total & & 280.35 & \\
\hline
\end{tabular}

\subsection{Infrared photometry}

In addition to the optical data, L. A. Crause obtained infrared JHKL measurements on the night of 2000 September 1 using the $0.75-\mathrm{m}$ telescope of the SAAO with the MkII infrared photometer (an upgraded version of the instrument described by Glass 1973). The data were reduced to the SAAO system by using standard stars defined by Carter (1990). As the infrared observations were obtained simultaneously with optical photometry, standard $B V I_{\mathrm{C}}$ JHKL magnitudes could be calculated (Table 4). The star was approaching a local minimum in its light curve when these measurements were taken.

These optical and infrared colours are consistent with the spectral type of the star as inferred from standard relations (Drilling \& Landolt 2000; Tokunaga 2000), but agree less well with the effective temperature determined from $u v b y \beta$ photometry. However, this may be due to the high amplitude of the light variations. The total colour amplitude implies temperature variations of $\approx 500 \mathrm{~K}$ between light extrema, sufficiently large to explain the apparent discrepancy.

\subsection{Spectroscopic observations}

Spectroscopic observations of HD 209295 were obtained with three different telescopes. We used the 1.9-m telescope at SAAO, the 1.9-m telescope at Mt. Stromlo (MS) in Australia, and the 1.2-m Euler telescope at the European Southern Observatory (ESO) in Chile. A summary of these measurements can be found in Table 5 .

The bulk of the data originated from SAAO using the GIRAFFE echelle fibre-fed spectrograph attached to the Cassegrain focus of the 1.9-m telescope. The GIRAFFE spectrograph has a resolving power of about 32000 . The $1024 \times 1024$ TEK CCD chip gives a resolution of 0.06-0.09 A per pixel. A ThAr arc lamp was used for wavelength calibration with arc spectra taken at regular intervals to calibrate possible drifts. The wavelength range was $4400-6680 \AA$, spread over 45 orders. Exposure times were normally $10 \mathrm{~min}$ for a signal-to-noise $(\mathrm{S} / \mathrm{N})$ ratio of about 30-60. A total of 422 spectra of HD 209295 was obtained during three observing runs.

The spectroscopic observations at MS were obtained with the coude echelle spectrograph on the Mount Stromlo 1.9-m telescope. Spectra with a resolution of $\sim 0.15 \AA$ at $\mathrm{H} \alpha$ were recorded on a $2048 \times 4096$ pixel TEK CCD. The wavelength range obtained with this set-up was $4500-6900 \AA$, spread over 42 orders. Exposure times were between $17.5-20 \mathrm{~min}$ for a $\mathrm{S} / \mathrm{N}$ ratio of about $25-60$. A ThAr arc lamp was used for wavelength calibration. As a result of poor weather, a total of only 15 spectra of HD 209295 was obtained.

The spectroscopic observations in Chile were performed with the Swiss 1.2-m Ritchey-Chretien Euler telescope at ESO, La Silla. Euler is equipped with a high-resolution echelle spectrograph, CORALIE, and a $2 \mathrm{k} \times 2 \mathrm{k}$ CCD camera with $15-\mu \mathrm{m}$ pixels. The resolving power amounts to 50000 and the total wavelength range is $3900-6800 \AA$ in 68 orders, without any gaps in the coverage. The CORALIE spectra are extracted on-line following a standard echelle reduction procedure. In the case of our measurements, wavelength calibration utilized the most recently obtained $\mathrm{ThAr}$ spectrum. Each order of the stellar spectrum was then divided by the blaze function. For a full description of the reduction scheme we refer the reader to Baranne et al. (1996). The integration times were adapted to the atmospheric conditions (seeing, presence of clouds) and ranged from about 15 to $18 \mathrm{~min}$. The typical $\mathrm{S} / \mathrm{N}$ ratio was $\approx 30$. In total, 38 spectra were obtained during one week. 


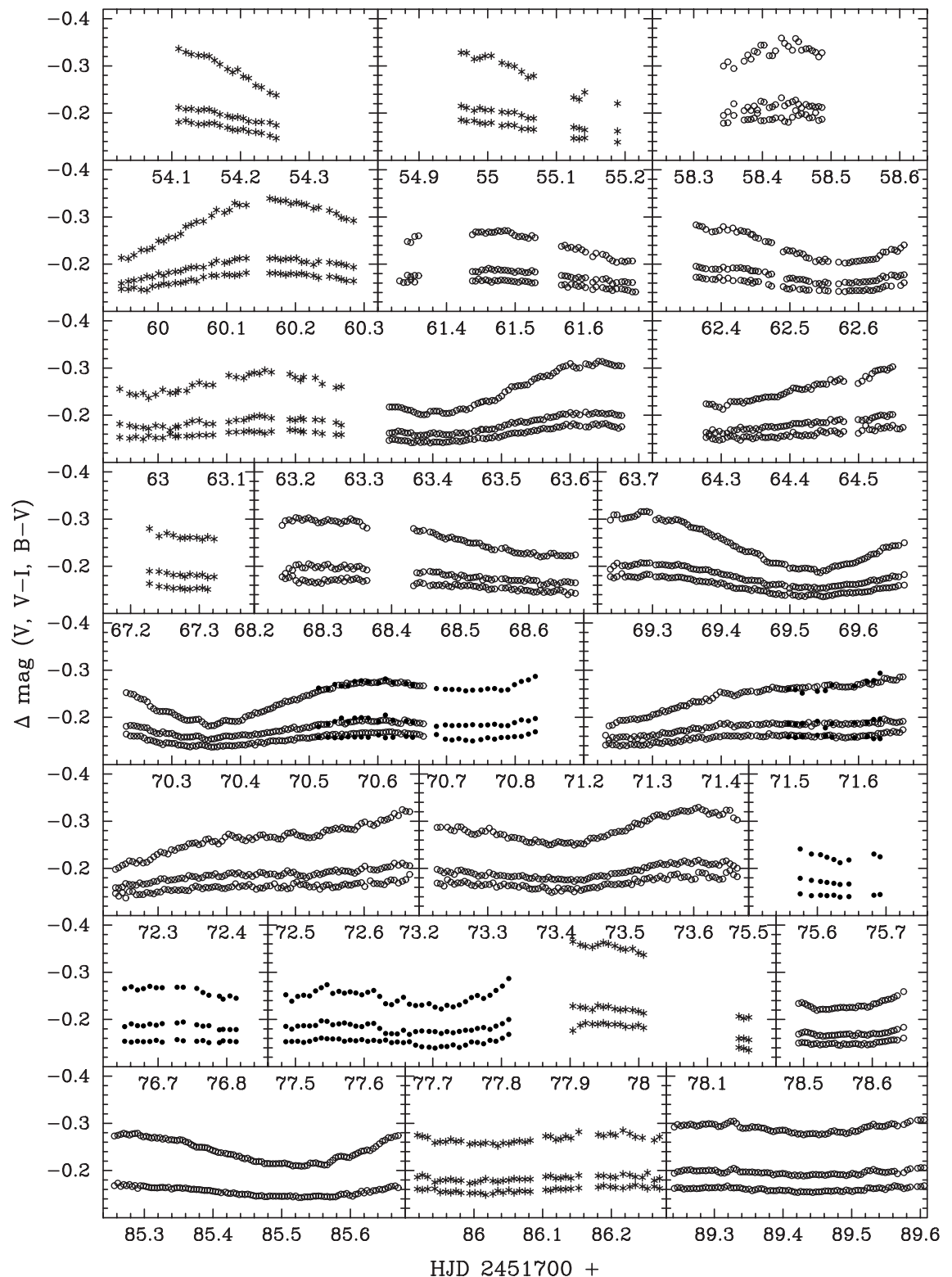

Figure 3. Light curves of HD 209295 obtained during the multisite campaign in 2000 August. The asterisks are measurements from SSO, the open circles those from SAAO and the filled circles are LNA data. The upper light curves in each panel are the $V$ data, the $\left(V-I_{\mathrm{C}}\right)$ variations are shown in the middle and the lowest are the $(B-V)$ light curves. Zero-points are relative to HD 209829; the $V$ zero-point is shifted by +0.11 mag for a better display. Note the multiperiodic slow and rapid light variability and the corresponding colour changes.

Table 4. Standard optical and infrared magnitudes of HD 209295 as measured on HJD 2451792.462

\begin{tabular}{lc}
\hline Filter & Magnitude \\
\hline$B$ & $7.623 \pm 0.005$ \\
$V$ & $7.370 \pm 0.005$ \\
$I_{\mathrm{C}}$ & $7.070 \pm 0.005$ \\
$J$ & $6.872 \pm 0.009$ \\
$H$ & $6.727 \pm 0.007$ \\
$K$ & $6.746 \pm 0.008$ \\
$L$ & $6.721 \pm 0.081$ \\
\hline
\end{tabular}

In contrast to the photometric observations, the spectroscopic measurements were not reduced centrally. For the SAAO data, we used the local reduction software (see http://www.saao.ac.za/ facilities/) for corrections for bias and flat-field, order extraction and wavelength calibration. The same reduction steps for the MS data were performed in IRAF.

\section{ANALYSIS}

\subsection{The photometry}

Our frequency analysis was performed with the programme PERIOD 98 (Sperl 1998). This package applies single-frequency power spectrum analysis and simultaneous multifrequency sine-wave fitting, but also has some advanced options which will be described 
Table 5. Log of the spectroscopic observations of HD 209295. The date with respect to HJD 2450000 , the run lengths, the mean integration times $t$ and the number of spectra, $N$, are given.

\begin{tabular}{|c|c|c|c|c|c|}
\hline Site & $\begin{array}{c}\text { Run start } \\
\text { HJD }-2450000\end{array}$ & $\begin{array}{l}\text { Length } \\
\text { (h) }\end{array}$ & $\begin{array}{c}t \\
(\mathrm{~s})\end{array}$ & $N$ & Observer \\
\hline SAAO & 1501.250 & 3.02 & 600 & 16 & LAB \\
\hline SAAO & 1502.268 & 2.39 & 600 & 13 & LAB \\
\hline SAAO & 1503.262 & 2.39 & 600 & 13 & LAB \\
\hline SAAO & 1504.249 & 2.99 & 600 & 16 & LAB \\
\hline MS & 1505.029 & 0.33 & 1200 & 1 & DJJ \\
\hline SAAO & 1505.255 & 2.42 & 600 & 13 & LAB \\
\hline SAAO & 1506.250 & 0.35 & 600 & 2 & LAB \\
\hline SAAO & 1507.297 & 1.29 & 600 & 7 & LAB \\
\hline SAAO & 1508.249 & 2.81 & 600 & 15 & LAB \\
\hline SAAO & 1509.251 & 2.54 & 600 & 12 & LAB \\
\hline MS & 1509.960 & 0.68 & 1200 & 3 & DJJ \\
\hline MS & 1510.924 & 2.22 & 1050 & 6 & DJJ \\
\hline SAAO & 1511.256 & 2.42 & 600 & 13 & LAB \\
\hline MS & 1511.942 & 1.62 & 1050 & 5 & DJJ \\
\hline SAAO & 1512.254 & 2.44 & 600 & 13 & LAB \\
\hline ESO & 1762.741 & 2.70 & 975 & 8 & TM \\
\hline ESO & 1763.700 & 3.69 & 1100 & 10 & TM \\
\hline SAAO & 1765.347 & 0.17 & 600 & 1 & $\mathrm{CK}$ \\
\hline ESO & 1765.744 & 3.69 & 1100 & 8 & TM \\
\hline ESO & 1766.703 & 2.92 & 1100 & 7 & TM \\
\hline SAAO & 1768.347 & 8.08 & 1095 & 21 & CK \\
\hline ESO & 1768.802 & 1.91 & 1100 & 5 & TM \\
\hline SAAO & 1769.336 & 8.21 & 1016 & 24 & CK \\
\hline SAAO & 1770.325 & 6.95 & 1000 & 21 & CK \\
\hline SAAO & 1771.345 & 8.12 & 975 & 24 & CK \\
\hline SAAO & 1772.345 & 7.70 & 1200 & 20 & $\mathrm{CK}$ \\
\hline SAAO & 1773.354 & 7.70 & 1000 & 23 & $\mathrm{CK}$ \\
\hline SAAO & 1774.406 & 1.34 & 1400 & 3 & CK \\
\hline SAAO & 1776.328 & 0.90 & 1500 & 2 & $\mathrm{CK}$ \\
\hline SAAO & 1777.388 & 1.23 & 1600 & 3 & CK \\
\hline SAAO & 1778.348 & 5.55 & 1557 & 7 & CK \\
\hline SAAO & 1849.267 & 2.42 & 600 & 12 & LAB \\
\hline SAAO & 1850.271 & 2.56 & 600 & 14 & LAB \\
\hline SAAO & 1851.274 & 2.50 & 600 & 10 & LAB \\
\hline SAAO & 1852.262 & 2.81 & 600 & 15 & LAB \\
\hline SAAO & 1853.258 & 2.68 & 600 & 14 & LAB \\
\hline SAAO & 1855.354 & 0.55 & 700 & 2 & LAB \\
\hline SAAO & 1856.258 & 2.44 & 600 & 13 & LAB \\
\hline SAAO & 1857.265 & 2.47 & 600 & 13 & LAB \\
\hline SAAO & 1858.264 & 2.25 & 600 & 12 & LAB \\
\hline SAAO & 1859.263 & 2.21 & 600 & 12 & LAB \\
\hline SAAO & 1860.265 & 2.23 & 600 & 12 & LAB \\
\hline SAAO & 1862.262 & 2.30 & 600 & 12 & LAB \\
\hline Total & & 128.19 & & 476 & \\
\hline
\end{tabular}

later as necessary. We calculated the spectral window and amplitude spectra of our data as well as the amplitude spectra of residual light curves after the previously identified periodicities had been removed using a multiperiodic fitting algorithm. Similar analyses were performed for all the three filters used. We adopted the mean frequencies for our final solution.

Our analysis was concentrated on multisite data from the year 2000. As a result of the different telescopes and detectors used, the 1999 data analysis is complicated by zero-point calibration problems. Also, the data runs were rather short in 1999 and the possibility exists that the star may have had a different amplitude at this time. We used the 1999 data only when the zero-point problems were relatively minor.

\subsubsection{The $\gamma$ Dor pulsations}

In Fig. 4 we show amplitude spectra of the $B$-filter data with

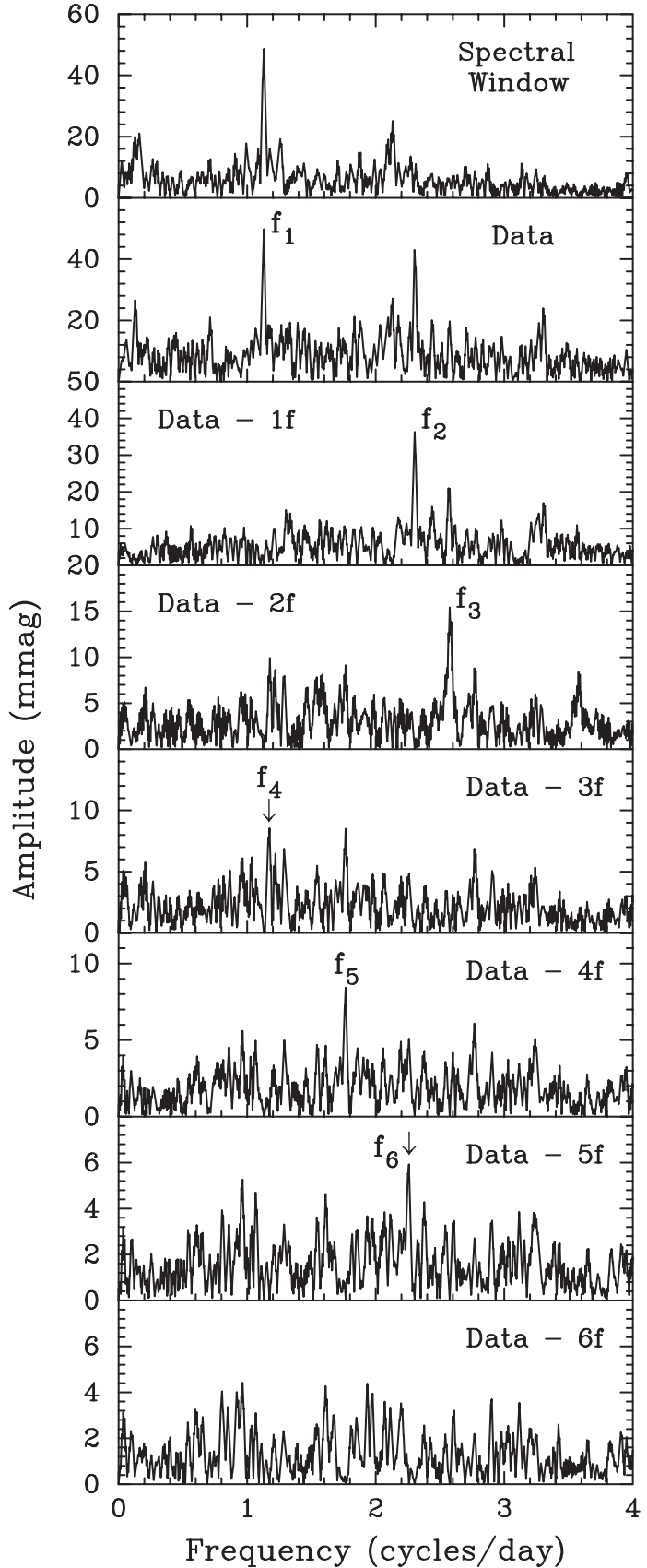

Figure 4. Top panel: Spectral window of the $B$-filter time-series photometry of HD 209295 obtained in the year 2000. The other panels show the amplitude spectra of the data with consecutive pre-whitening. Six significant periodicities are present.

consecutive pre-whitening by the low frequencies. For the 2000 data we calculated the window function as the Fourier transform of a single sinusoid with frequency 1.129 cycle $^{-1}$ and amplitude of $50 \mathrm{mmag}$. Because of the multisite coverage, aliasing is not a problem. We detected six significant frequencies in the light variations. The first four frequencies were already shown in Table 1.

The significance of a detection was estimated using the $\mathrm{S} / \mathrm{N}$ ratio criterion of Breger et al. (1993). The residuals after pre-whitening this frequency solution suggest that more periodicities are present. The highest peaks in the residual spectra are located at the same frequencies in each of the $B, V$, and $I_{\mathrm{C}}$ data sets, and the "noise 
Table 6. Results of a multifrequency solution for HD 209295 derived from the photometric data. Frequency error estimates range from \pm 0.0001 cycle $^{-1}$ to \pm 0.002 cycle d $^{-1}$, for the strongest and weakest $\gamma$ Dor mode, respectively, and \pm 0.005 cycle $^{-1}$ for the $\delta$ Sct mode. Signalto-noise ratios following Breger et al. (1993), calculated from the $B$ filter data from the year 2000, are also given. A $S / N>4$ is taken to be a significant detection of a signal.

\begin{tabular}{|c|c|c|c|c|c|c|c|}
\hline \multirow[b]{2}{*}{ ID } & \multirow[b]{2}{*}{$\begin{array}{l}\text { Frequency } \\
\qquad\left(\mathrm{d}^{-1}\right)\end{array}$} & \multirow[b]{2}{*}{$S / N$} & \multicolumn{3}{|c|}{ Measurements from the year 2000} & \multicolumn{2}{|c|}{ Measurements from the year 1999} \\
\hline & & & $\begin{array}{c}B \text { amplitude } \\
\text { (mmag) } \\
\pm 0.4\end{array}$ & $\begin{array}{c}V \text { amplitude } \\
\text { (mmag) } \\
\pm 0.3\end{array}$ & $\begin{array}{c}I \text { amplitude } \\
\text { (mmag) } \\
\pm 0.2\end{array}$ & $\begin{array}{c}B \text { amplitude } \\
\text { (mmag) } \\
\pm 0.6\end{array}$ & $\begin{array}{c}V \text { amplitude } \\
\text { (mmag) } \\
\pm 0.5\end{array}$ \\
\hline \multicolumn{8}{|c|}{$\gamma$ Dor frequencies } \\
\hline$f_{1}$ & 1.1296 & 35.9 & 49.6 & 38.5 & 23.0 & 35.9 & 26.9 \\
\hline$f_{2}$ & 2.3024 & 28.3 & 39.1 & 28.3 & 15.5 & 24.5 & 17.3 \\
\hline$f_{3}$ & 2.5758 & 11.6 & 16.0 & 11.4 & 6.2 & 21.7 & 15.8 \\
\hline$f_{4}$ & 1.1739 & 9.0 & 12.4 & 7.9 & 4.6 & 5.4 & 4.9 \\
\hline$f_{5}$ & 1.7671 & 7.0 & 9.7 & 7.4 & 4.1 & 11.0 & 8.5 \\
\hline$f_{6}$ & 2.2572 & 5.4 & 7.4 & 5.3 & 3.1 & $\mathrm{n} / \mathrm{a}$ & $\mathrm{n} / \mathrm{a}$ \\
\hline \multicolumn{8}{|c|}{$\delta$ Sct frequency } \\
\hline$f_{A}$ & 25.9577 & 5.3 & 1.8 & 1.4 & 0.8 & 1.5 & 1.3 \\
\hline
\end{tabular}

level' decreases from $B$ to $I_{\mathrm{C}}$. This suggests that most of the 'noise' in the blue may well be due to additional periodicities. The derived frequencies and amplitudes are listed in Table 6.

\subsubsection{The $\delta$ Sct pulsations}

The zero-point uncertainties in the data set from 1999 have little effect in the frequency range in which the $\delta$ Sct pulsations are present. Consequently, we can incorporate these observations into the frequency analysis. We therefore pre-whitened the data by the six-frequency solution discussed above from the 2000 data. For the 1999 data we pre-whitened by the first five of these frequencies which were found in common with the analysis of the 2000 data. This pre-whitening is important because low-frequency variations can artificially increase the noise level in the high-frequency domain through spectral leakage. The amplitude spectrum of the combined $B$-filter data and subsequent pre-whitening are shown in Fig. 5.

Although several peaks in the amplitude spectra in Fig. 5 are prominent, only one signal is a significant detection. This is surprising, as typical multiperiodic beating is seen in the light curves of Fig. 3, e.g. HJD 2451770.35 versus HJD 2451770.55. We therefore checked whether these signals could be non-coherent by analysing several subsets of data. No evidence for short-lived $\delta$ Sct type variations or variations with changing amplitude was found. In fact, several of the more conspicuous signals were present in all data subsets (e.g. in different subsets of the multisite data or in individual filters). Thus we suspect that several such periodicities may be real. A multifrequency solution with $f_{A}$ and the three next strongest variations in the $\delta$ Sct regime fits the shortterm variations reasonably. The corresponding peaks are marked with arrows in the lowest panel of Fig. 5. The frequency range of possibly excited $\delta$ Sct pulsations can be constrained to $10-32$ cycle $\mathrm{d}^{-1}$. We list the results of our frequency analysis of the photometric data in Table 6.

We need to make two remarks here. First, the results for the data from 1999 are only listed for completeness in Table 6. We suspect that the amplitudes of the $\gamma$ Dor pulsations in this data set are artificially decreased not only because of zero-point problems, but also because of poor data sampling. Secondly, we stress that these

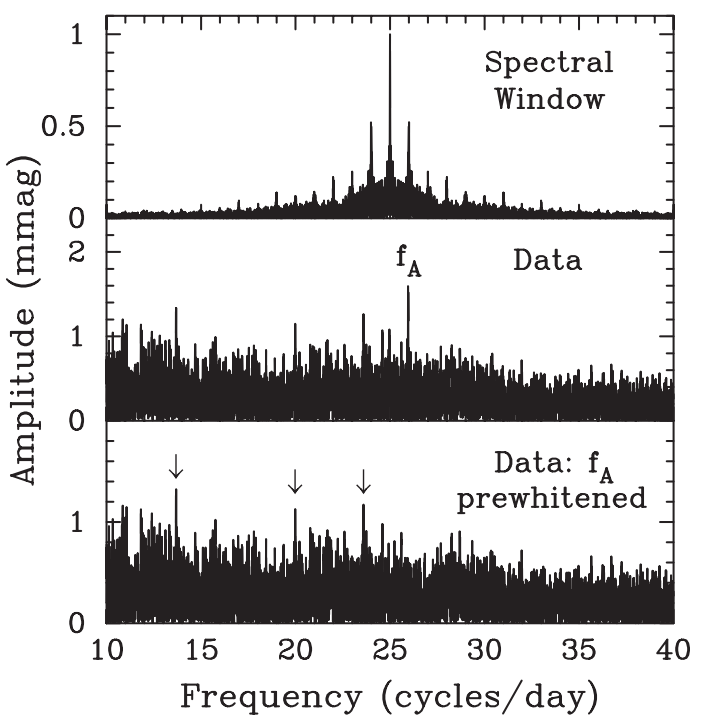

Figure 5. The spectral window of the combined residual $B$-filter data and amplitude spectra in the frequency domain of the $\delta$ Sct pulsations. Only one frequency is convincingly detected, but the presence of several low-

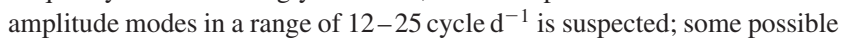
further frequencies are indicated.

are only preliminary results. We will revisit the frequency analysis in Section 3.4.

\subsection{Spectroscopic analyses}

The first step in the analysis is to rectify the spectra, i.e. to place the continuum. This was done by using an unbroadened synthetic spectrum with $T_{\text {eff }}=7500 \mathrm{~K}, \log g=4.00$ as a template, using the SPECTRUM code (Gray \& Corbally 1994). A running median of each echelle order was divided by the corresponding section of the synthetic spectrum and taken to represent the response function of the instrument. A polynomial of degree 5 was fitted to the response function and used to correct the observed spectrum. The result is the rectified spectrum of the star used for cross-correlation. 


\subsubsection{Radial velocities}

For each order, the observed rectified spectrum was correlated with the corresponding section of the synthetic spectrum after removing the unit continuum. The resulting correlation function is, in effect, the mean line profile with the continuum removed. The 'radial velocity' for each order is obtained by fitting a quadratic to the correlation function and finding the position of the maximum. The mean radial velocity from all the orders is also obtained. A standard error of typically $1-3 \mathrm{~km} \mathrm{~s}^{-1}$ was found for the SAAO spectra.

A few of the spectroscopic observations listed in Table 5 turned out to be of a quality too poor for further use. Four spectra were discarded, and 472 were retained. Some slight deviations $\left(<0.5 \mathrm{~km} \mathrm{~s}^{-1}\right)$ in the seasonal radial velocity zero-points in the SAAO spectra were rectified by means of telluric lines.

An immediate finding from our time-resolved spectroscopy is that the radial velocities of HD 209295 are strongly variable. The total radial velocity amplitude is in excess of $100 \mathrm{~km} \mathrm{~s}^{-1}$, which cannot be due to the pulsations of the star. HD 209295 must be a member of a binary system.

We therefore attempted to determine the orbital period. Visual inspection of the radial velocity curve suggests $P_{\text {orb }} \sim 3 \mathrm{~d}$, and Fourier analysis (upper panel of Fig. 6) indeed implies an orbital frequency near 0.32 cycle $^{-1}$, but our data set is affected by aliasing. Pre-whitening trial frequencies suggested that the shape of the orbital radial velocity curve is not sinusoidal.

This is a situation in which use of the residualgram method (Martinez \& Koen 1994) is indicated, which is based on a
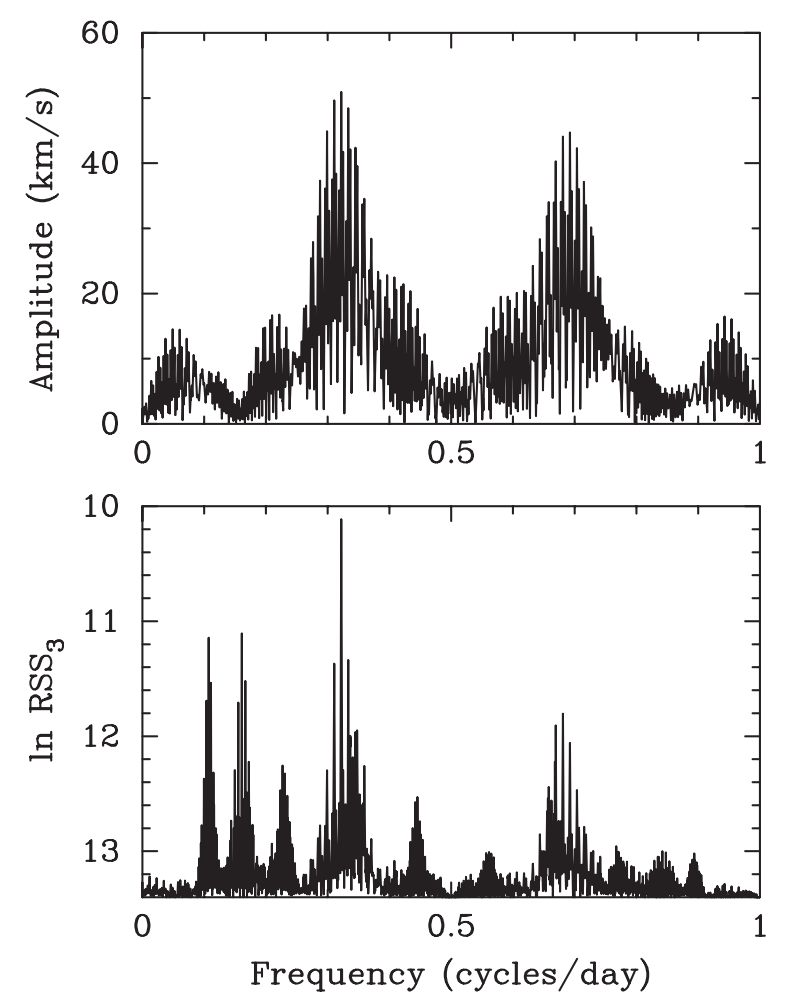

Figure 6. Upper panel: Fourier amplitude spectrum of the radial velocities of HD 209295. An orbital period near $3 \mathrm{~d}$ is implied, but severe aliasing is present. Lower panel: residual sum of squares spectrum of a three-harmonic fit $\left(\mathrm{RSS}_{3}\right)$ to the radial velocities. Although some power leaks into subharmonics, the correct orbital frequency is now unambiguously detected. least-squares fit of a sine wave with $M$ harmonics. The residual sum of squares at each trial frequency is evaluated; $M$ can be chosen freely. In that way, alias ambiguities can be circumvented by taking advantage of the information in the Fourier harmonics. Preliminary trials with Fourier analysis suggested that $M=3$ is a good choice for a residualgram, as shown in the lower panel of Fig. 6.

The residualgram method eliminated the aliasing problem for the determination of the orbital frequency. We use its result for a refinement of the orbital frequency by using PERIOD 98 (Sperl 1998). This leads to an improved orbital frequency of $f_{\text {orb }}=$ 0.32198 cycle d $^{-1}\left(P_{\text {orb }}=3.10575 \mathrm{~d}\right)$.

To determine the orbital solution from our radial velocities, we first used the SAAO data only, which are the most extensive (almost 90 per cent of all spectra) and are homogeneous. We weighted the individual measurements based on their standard errors. Measurements with standard errors smaller than $2 \mathrm{~km} \mathrm{~s}^{-1}$ were given a weight of 1 ; the weights decreased down to 0.2 for a few measurements with standard errors between $8-9 \mathrm{~km} \mathrm{~s}^{-1}$. We determined an initial orbital solution from these data with an updated version (Strassmeier, private communication) of the differential-correction method (Barker, Evans \& Laing 1967).

Using the initial parameters from these methods, we examined the relative zero-points of the three blocks of SAAO measurements; no third body in the system was detected. We then fitted all the campaign data with these orbital parameters and examined the zero-points of the measurements from the other sites. The ESO radial velocities needed to be shifted by $+7 \mathrm{~km} \mathrm{~s}^{-1}$. We then

Table 7. The orbital solution from radial velocities of HD 209295. The symbols have their usual meanings; $\omega$ is the argument of periastron.

\begin{tabular}{lcc}
\hline Parameter & Unit & Value \\
\hline$P_{\text {orb }}$ & $(\mathrm{d})$ & $3.10575 \pm 0.00010$ \\
$\gamma$ & $\left(\mathrm{km} \mathrm{s}^{-1}\right)$ & $-23.7 \pm 0.4$ \\
$K$ & $\left(\mathrm{~km} \mathrm{~s}^{-1}\right)$ & $54.2 \pm 0.7$ \\
$e$ & & $0.352 \pm 0.011$ \\
$\omega$ & $\left({ }^{\circ}\right)$ & $31.1 \pm 2.0$ \\
$T_{0}$ & $(\mathrm{HJD})$ & $2451771.864 \pm 0.014$ \\
$a_{1} \sin i$ & $\left(\mathrm{R}_{\odot}\right)$ & $3.11 \pm 0.04$ \\
$f(m)$ & $\left(\mathrm{M}_{\odot}\right)$ & $0.042 \pm 0.002$ \\
\hline
\end{tabular}

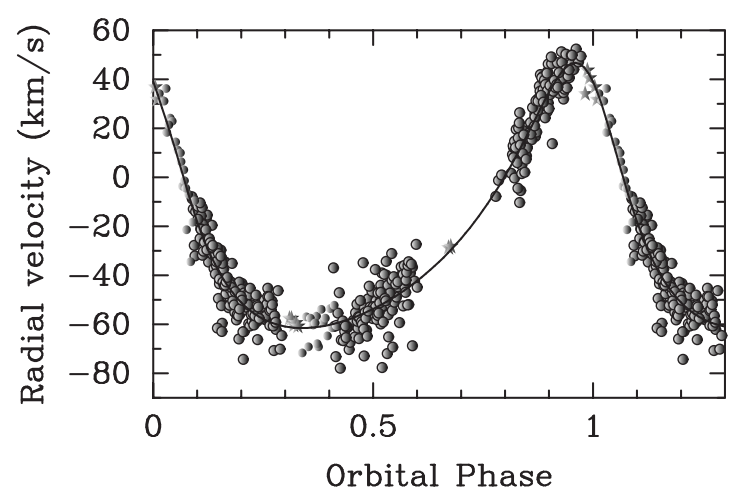

Figure 7. Radial velocities of HD 209295 phased with the orbital solution (solid line). Open circles are SAAO radial velocities, filled circles are ESO radial velocities and star symbols denote the MS measurements. We note that although the number of spectra from ESO and MS is small, they cover important phases of the orbit and are therefore quite valuable. The formal radial velocity errors quoted in Section 3.2.1 underestimate the true errors. 

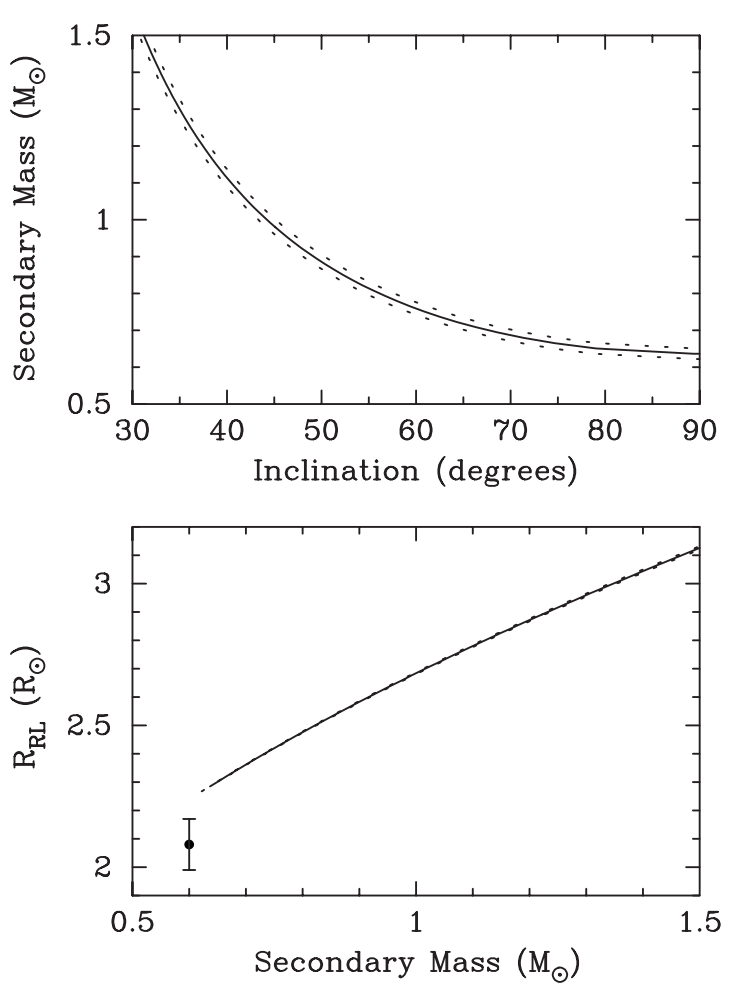

Figure 8. Upper panel: the mass of the companion of HD 209295 versus orbital inclination. The solid line is for a primary mass of $1.84 \mathrm{M}_{\odot}$ as inferred in Section 1.1, the dotted lines represent the $1 \sigma$ limits of this determination. Lower panel: the Roche Lobe radius of the HD 209295 primary at periastron depending on secondary mass, again for $M=1.84 \pm 0.07 \mathrm{M}_{\odot}$. The filled circle with the error bar shows our radius determination for HD 209295 from Section 1.1.

calculated our final solution from all the data, with the mean radial velocity fixed. This orbital solution is shown in Table 7 and Fig. 7.

We note that an extrapolation of our orbital solution to the measurements of Grenier et al. (1999) quoted in the introduction results in a good fit. The two measurements by these authors were taken at similar orbital phases, which explains the comparably small radial velocity difference.

Having solved for the orbital parameters, we can now discuss two issues: the nature of the companion and the possibility of mass transfer in the system. In the upper panel of Fig. 8, we show the dependence of the secondary mass on orbital inclination for various values of the primary mass. From this, we see that $M_{2}>0.62 \mathrm{M}_{\odot}$. The lower panel of Fig. 8 shows that HD 209295 may come close to filling its Roche Lobe (calculated with the approximation by Eggleton 1983) at periastron if the companion has a small mass. However, we will show in Section 4.1 that the companion is probably too massive for the primary to fill its Roche Lobe.

Assuming that the companion of HD 209295 is a main-sequence star, its absolute magnitude $M_{\mathrm{v}}>4.4$; otherwise its spectral lines would be detected. A spectral type of G0 and later is therefore suggested with a mass $<1.05 \mathrm{M}_{\odot}$ (Drilling \& Landolt 2000). Our photometry also shows no eclipses (within a conservative limit of $4 \mathrm{mmag}$ ), allowing us to infer the constraint $i<80^{\circ}$. A mainsequence companion of $0.62<M_{2}<1.05 \mathrm{M}_{\odot}$ should be detected by means of its infrared excess. We will examine this in Section 3.5.

After removal of the orbital variation, the residual radial

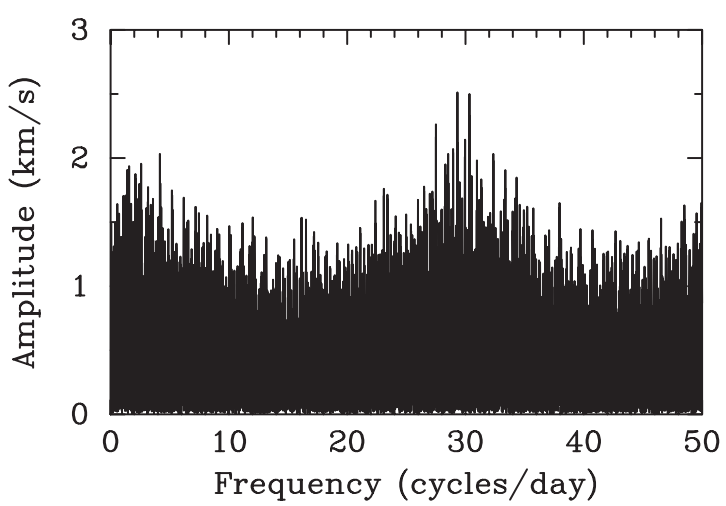

Figure 9. The amplitude spectrum of the residual radial velocities of HD 209295 after removing the orbital solution.

velocities can be searched for pulsational signals. The amplitude spectrum of these residuals can be found in Fig. 9. Although there are signals in the same frequency range as in the photometry, none give a reliable detection according to the signal-to-noise ratio criterion of Breger et al. (1993). Consequently, we need to continue the examination of the spectroscopic evidence of the pulsations of HD 209295 by looking at the line-profile variations.

\subsubsection{Projected rotational velocity}

The correlation profiles for each season can be co-added (after correcting with our orbital solution) to form what is essentially a mean line profile. The projected rotational velocity, $v \sin i$, can be determined by fitting a model profile of a rotating star. The model is spherical with a linear limb darkening coefficient $u=0.3$ and the visible hemisphere is divided into a large number of surface elements. The intrinsic line profile for each element summed over the hemisphere, weighted according to limb darkening, is computed. The projected rotational velocity and mean radial velocity are adjusted until a best fit to the observed profile is obtained. We obtain $v \sin i=108 \pm 10 \mathrm{~km} \mathrm{~s}^{-1}$ for the SAAO measurements from 1999 and the second set from 2000, and $v \sin i=98 \pm 10 \mathrm{~km} \mathrm{~s}^{-1}$ for the first data set of the year 2000 . Because of the uncertainty in the wings of the correlation profile, the fit was performed using the part of the profile within $90 \mathrm{~km} \mathrm{~s}^{-1}$ of the line centre.

\subsubsection{The high-order profile variations}

In a recent paper on the $\delta$ Sct star $38 o^{1}$ Eri, Balona (2000) was able to show that pulsation modes of high spherical degree $\ell$ are easy to resolve in stars with moderate to high rotational velocities. Bearing in mind the result from the previous paragraph, HD 209295 falls within this category. We will only use the SAAO spectra for the following analysis, as these are the most homogeneous and best sampled.

Line profile variations due to modes of high degree are seen as moving subfeatures in the correlation profiles of the spectra used to determine the radial velocities. As a particular subfeature crosses the centre of the profile, it determines the radial velocity. A short while later another travelling subfeature will appear and the radial velocity will suddenly change. These discontinuous jumps are partly responsible for the rather high errors of the radial velocities.

From the spacing of the travelling subfeatures, we estimate that the spherical degree is $\ell \approx 5$. To enhance the profile variations, we 

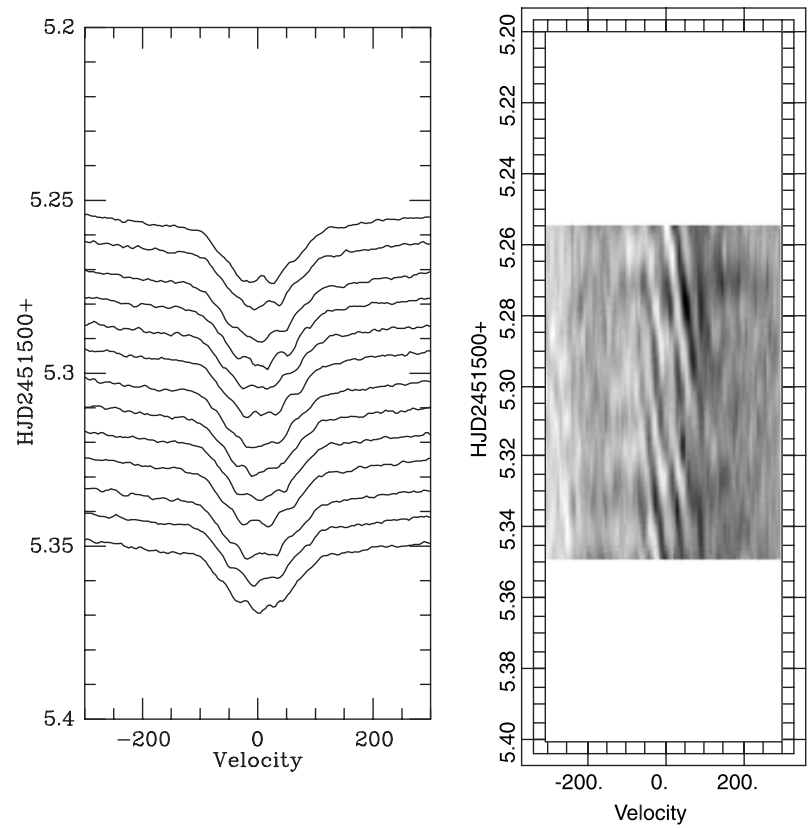

Figure 10. Left-hand panel - correlation function; right-hand panel - greyscale difference after removing the correlation function. The data are from the 1999 season. The abscissa is in $\mathrm{km} \mathrm{s}^{-1}$ and the ordinate is the HJD measured from HJD 2451500.000 .
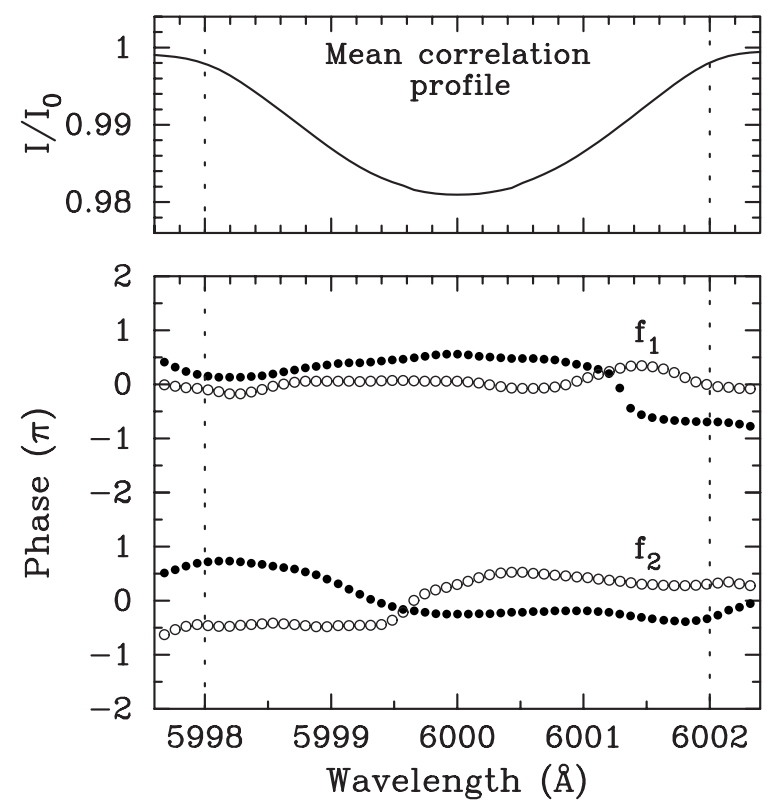

Figure 11. Phase diagram for two modes of HD 209295 (lower panel) through the correlation profile (upper panel), which is arbitrarily centred at $6000 \AA$. The full circles denote the phases of the mode frequency, and the open circles that of its first harmonic. The zero-points in the phases are arbitrary. The dotted vertical lines indicate $v \sin i$, as determined in Section 3.2.2, translated into units of wavelength. This is also the position in the correlation profile where we determine the total phase change.

removed the orbital motion and constructed the mean correlation profile from the SAAO data. Each correlation profile was divided by the mean profile to construct 'difference' profiles at the given times. The moving features are most clearly seen when these difference profiles are arranged in a time sequence and converted to a grey-scale image. An example is shown in Fig. 10.
Table 8. The total phase variation of the mode frequencies $\Delta \phi$, their first harmonics $\Delta \phi_{1}$, and the implied mode identifications following Telting \& Schrijvers (1997).

\begin{tabular}{ccccc}
\hline ID & $\begin{array}{c}\Delta \phi_{0} \\
(\pi \mathrm{rad})\end{array}$ & $\begin{array}{c}\Delta \phi_{1} \\
(\pi \mathrm{rad})\end{array}$ & $\ell$ & $|m|$ \\
\hline$f_{1}$ & 0.85 & -0.05 & 1 & 1 \\
$f_{2}$ & 1.06 & -0.77 & 1 & 1 \\
\hline
\end{tabular}

While it is clear from Fig. 10 that periodic high-order line profile variations are present, it is not possible to determine the frequencies from the radial velocities alone, as shown before. Clearly, the radial velocity is a poor indicator for modes of high degree.

A common method to determine periodicities in line profile variations is to calculate the periodogram along wavelength bins throughout the line profile, or across the correlation profile. Most of the signal in this analysis is located at frequencies very close to the orbital period and its aliases, suggesting that the removal of the orbital variation did not work satisfactorily. However, we found the signatures of two previously detected modes in the line profiles, although they cannot be detected without prior knowledge. These are the photometric $\gamma$ Doradus modes $f_{1}$ and $f_{2}$.

This finding gives us the possibility to attempt a mode identification with the method proposed by Telting \& Schrijvers (1997). This technique utilizes the phase change of a mode as it progresses through the line profile, and provides some simple mode identification diagnostics depending on the total phase change of the mode frequency and its first harmonic (e.g. $\Delta \phi_{0} \approx \pi \ell$ ). We calculated these phase changes for the two modes which we think are present and we show their behaviour through the correlation profile in Fig. 11. The mode identification implied by this analysis is summarized in Table 8.

We note that Telting \& Schrijvers (1997) have given error estimates for the mode identifications with this method with \pm 1 for $\ell$ and \pm 2 for $m$. The identifications are plausible: $f_{1}$ and $f_{2}$ have high photometric amplitude.

\subsection{Combining the spectroscopic and photometric information}

Comparing the frequencies of the $\gamma$ Dor pulsations in Table 6 to the orbital frequency we see that the photometric mode $f_{3}$ corresponds exactly to $8 f_{\text {orb }}$, and $f_{6}$ is, within the errors, consistent with $7 f_{\text {orb. }}$. As noted in Section 1.1, the relation $f_{4}=f_{2}-f_{1}$ is also present within the mode frequencies.

Consequently, we revisited the frequency analysis of the photometric data. We again restricted ourselves to the data from 2000. This time, however, we used PERIOD 98's capability to fix signal frequencies to certain values, in our case integer multiples of the orbital frequency, and to perform non-linear least-squares fits with frequencies thus fixed.

We started by using the six $\gamma$ Dor frequencies in Table 6 , and proceeded by the usual pre-whitening procedure. The corresponding amplitude spectra can be found in Fig. 12; they are generated by combining the $B$-filter residuals with the $V$-filter residuals multiplied by a factor of 1.29 .

The highest-amplitude peak in the upper panel of Fig. 12 does, indeed, correspond to $3 f_{\text {orb }}$. In addition $5 f_{\text {orb }}$ (second panel of 


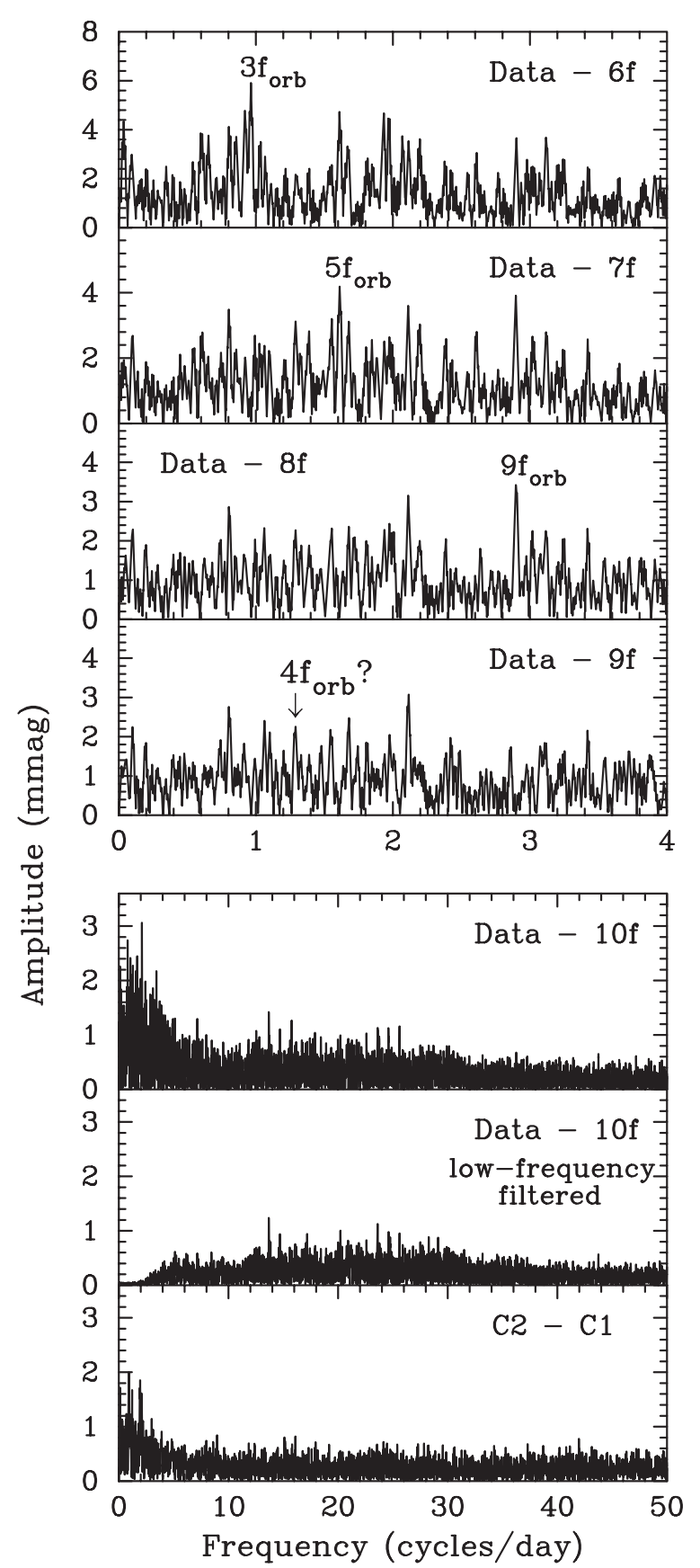

Figure 12. Top panel: the amplitude spectrum of the combined $B$ and scaled $V$-filter residuals of HD 209295 after subtraction of the frequency solution of Table 6. Another harmonic of the orbital frequency is detected. In the following three panels we show the effects of further pre-whitening. All new detections are again harmonics of $f_{\text {orb }}$. Third panel from bottom: the residual amplitude spectrum in a wider frequency range. Second panel from bottom: the residual amplitude spectrum after filtering the residual lowfrequency variations. Bottom panel: amplitude spectrum of the differential magnitudes of the comparison stars.

Fig. 12) and $9 f_{\text {orb }}$ (third panel of Fig. 12) are also found. This latter frequency corresponds to exactly $f_{1}+f_{5}$. We therefore fixed this frequency combination to its exact value before proceeding. After pre-whitening these frequencies, some peaks, including one at $4 f_{\text {orb }}$, are still visible in each filter, but not at a level considered significant.
The third panel from the bottom of Fig. 12 shows the residual amplitude spectra in a wider frequency range after pre-whitening by the $\delta$ Sct mode, $f_{A}$. Again, there is evidence of additional $\gamma$ Dor and weak $\delta$ Sct periodicities. The referee suggested to filter out the slow variability to examine the claimed $\delta$ Sct variability further. This is done in the second panel from the bottom of Fig. 12; albeit somewhat affected by the filtering, the additional $\delta$ Sct variations remain with similar amplitudes. They are therefore not artefacts from spectral leakage of the low frequencies. In the lowest panel of Fig. 12 we show the amplitude spectrum of the comparison star data. Although the number of observations of each comparison star is half that of the program star, the noise level in this periodogram is lower still, again suggestive of further periodicities below the detection level.

In Table 9 we show the final multifrequency solution for the 2000 data set. The corresponding results for 1999 are of little value, as the data sampling is too poor to attempt such a fit. Error estimates are formal values following Montgomery \& O'Donoghue (1999). While they should be quite realistic for the $\delta$ Sct modes, they probably underestimate the errors for the $\gamma$ Dor modes by a factor of $3-4$.

The rms residual errors per single data point for this solution are $7.0 \mathrm{mmag}$ in the $B$ filter, $5.7 \mathrm{mmag}$ in the $V$ filter and $4.5 \mathrm{mmag}$ in the $I$ filter. These values again suggest undetected periodicities, because the rms scatter of a single comparison star measurement is $5.1 \mathrm{mmag}$ in $B, 4.0 \mathrm{mmag}$ in $V$ and $4.2 \mathrm{mmag}$ in $I$.

Another estimate of the orbital period can be made from the frequency analysis. We find $P_{\text {orb }}=3.1057 \pm 0.0010$, consistent with the determination in Section 3.2.1. We have examined the residual light curve for possible ellipsoidal variations, but found none exceeding $2 \mathrm{mmag}$. Finally, one might suspect that $f_{6}=2 f_{1}$, but a fit assuming this relation gives a significantly poorer solution than the one listed in Table 9.

\subsection{Attempts at mode identification from colour photometry}

The main reason for observing HD 209295 in more than one filter was to attempt a mode identification. This method relies on comparing theoretical amplitude ratios and phase differences of the variations in different wavebands with the observed values. The high amplitude of the light variations of HD 209295 makes the star very well suited for this method. Because of the very low amplitude of the $\delta$ Sct pulsations, only the $\gamma$ Dor modes are considered. However, our calculations indicate that the amplitude ratios and phase differences for the $\delta$ Sct mode are consistent with pulsation.

We first attempted to apply the method developed by Koen et al. (1999) to the $\gamma$ Dor modes. This technique uses the observed amplitude ratios and phase differences in all filters simultaneously. Unfortunately, no meaningful results were obtained. We then constructed two-colour diagnostic diagrams showing amplitude ratio as a function of phase difference (Watson 1988). In such diagrams, the theoretically determined areas of interest are specified and compared with the observations. We used the Warsaw-New Jersey stellar evolution and W. A. Dziembowski's NADROT pulsation code for models of 1.8 and $1.9 \mathrm{M}_{\odot}$ with effective temperatures between 7250 and $7800 \mathrm{~K}$. These models span the possible range of parameters for HD 209295. We then computed theoretical amplitude ratios and phase shifts for the eigenmodes of those models following Balona \& Evers (1999) and examined their location in the corresponding diagrams. We noticed that (except for a few combinations of $Q$ and $\ell$ ) the results clustered in welldefined regions depending on $\ell$. Hence, we defined those regions 
Table 9. The final multifrequency solution for HD 209295 from all the year 2000 photometry. Pulsational phases for mean light level are given with respect to a time of periastron passage, HJD 245 1771.864. Amplitude signal-to-noise ratios are calculated from Fig. 12.

\begin{tabular}{|c|c|c|c|c|c|c|c|c|c|}
\hline ID & Combination & $\begin{array}{l}\text { Frequency } \\
\left(\mathrm{d}^{-1}\right)\end{array}$ & $S / N$ & $\begin{array}{c}\text { B Ampl. } \\
(\mathrm{mmag}) \\
\pm 0.3\end{array}$ & $\begin{array}{c}B \text { phase } \\
\left({ }^{\circ}\right)\end{array}$ & $\begin{array}{c}\text { VAmpl. } \\
(\mathrm{mmag}) \\
\pm 0.2\end{array}$ & $\begin{array}{c}V \text { phase } \\
\left({ }^{\circ}\right)\end{array}$ & $\begin{array}{l}\text { I Ampl. } \\
(\mathrm{mmag}) \\
\pm 0.2\end{array}$ & $\begin{array}{c}I \text { phase } \\
\left({ }^{\circ}\right)\end{array}$ \\
\hline \multicolumn{10}{|c|}{$\gamma$ Dor frequencies } \\
\hline $\begin{array}{l}f_{1} \\
f_{2} \\
f_{3} \\
f_{4} \\
f_{5} \\
f_{6} \\
f_{7} \\
f_{8} \\
f_{9}\end{array}$ & $\begin{array}{c}8 f_{\text {orb }} \\
f_{2}-f_{1} \\
9 f_{\text {orb }}-f_{1} \\
7 f_{\text {orb }} \\
3 f_{\text {orb }} \\
5 f_{\text {orb }} \\
9 f_{\text {orb }}\end{array}$ & $\begin{array}{l}1.12934 \pm 0.00005 \\
2.30217 \pm 0.00006 \\
2.57593 \pm 0.00011 \\
1.17283 \pm 0.00004 \\
1.76859 \pm 0.00005 \\
2.25394 \pm 0.00011 \\
0.96597 \pm 0.00011 \\
1.60996 \pm 0.00011 \\
2.89792 \pm 0.00011\end{array}$ & $\begin{array}{r}63.7 \\
49.9 \\
23.2 \\
14.4 \\
13.6 \\
10.6 \\
8.9 \\
5.8 \\
5.7\end{array}$ & $\begin{array}{r}50.3 \\
39.4 \\
18.3 \\
11.4 \\
10.8 \\
8.4 \\
7.0 \\
4.6 \\
4.5\end{array}$ & $\begin{aligned}-39.4 & \pm 0.3 \\
-115.4 & \pm 0.4 \\
66.5 & \pm 0.8 \\
-80.4 & \pm 1.3 \\
96.5 & \pm 1.3 \\
2.0 & \pm 1.7 \\
-39.2 & \pm 2.0 \\
-162.1 & \pm 3.1 \\
47.0 & \pm 3.2\end{aligned}$ & $\begin{array}{r}38.9 \\
28.7 \\
13.2 \\
7.6 \\
8.2 \\
6.6 \\
6.2 \\
3.9 \\
3.5\end{array}$ & $\begin{array}{r}-40.3 \pm 0.3 \\
-116.4 \pm 0.4 \\
67.2 \pm 0.9 \\
-73.3 \pm 1.5 \\
95.4 \pm 1.4 \\
-1.0 \pm 1.8 \\
-35.9 \pm 1.9 \\
-158.6 \pm 3.0 \\
48.9 \pm 3.3\end{array}$ & $\begin{array}{r}23.5 \\
15.4 \\
7.3 \\
4.0 \\
4.8 \\
3.5 \\
4.9 \\
1.9 \\
2.2\end{array}$ & $\begin{aligned}-42.6 & \pm 0.4 \\
-117.0 & \pm 0.6 \\
64.3 & \pm 1.3 \\
-68.5 & \pm 2.4 \\
86.2 & \pm 2.0 \\
2.0 & \pm 2.8 \\
-33.3 & \pm 1.9 \\
-173.9 & \pm 5.2 \\
53.5 & \pm 4.3\end{aligned}$ \\
\hline \multicolumn{10}{|c|}{$\delta$ Sct frequency } \\
\hline$f_{A}$ & $f_{\text {orb }}$ & $\begin{array}{l}25.9577 \pm 0.0015 \\
0.32199 \pm 0.00011\end{array}$ & 5.2 & 1.8 & $158.9 \pm 8.3$ & 1.4 & $162.8 \pm 8.3$ & 0.8 & $179.1 \pm 12.2$ \\
\hline
\end{tabular}

as our regions of interest and compared the observations to them. An example is shown in Fig. 13.

Before evaluating the potential for mode identifications from Fig. 13, two comments need to be made. First, we only show the results for the $B$ and $I_{\mathrm{C}}$ filter pair, as the diagrams are similar in appearance for the other two possible filter combinations, but the observational errors are larger.

Secondly, the locations of the areas of interest depend quite strongly on the pulsation constant $Q$. This is a well-known result (e.g. discussed by Garrido 2000), and is the reason why we have separated the results into three subsets which depend on $Q$. We chose a suitable subdivision based on the observed $\gamma$ Dor frequencies.

The implications from Fig. 13 can be summarized as follows. For modes with $Q>0.2 \mathrm{~d}$, the mode identification method gives meaningless results. For the modes with $Q<0.2 \mathrm{~d}$ there is some agreement, but this might be coincidence. In any case, the amplitude ratios of the photometric modes are consistent with $\ell=1$ or 2 , but not with $\ell=3$.

\subsection{The infrared colours and the companion of HD 209295}

In Section 3.2.1 we have shown that the binary companion of HD 209295 must have a mass of at least $0.6 \mathrm{M}_{\odot}$. If it were a mainsequence star, this would correspond to a spectral type of K5. The most luminous possible main-sequence companion to HD 209295 is a G0 star. A star more luminous than this would be detected in the spectrum. We decided to search for a companion in the infrared. For this purpose we show of the system optical and infrared colours in Table 4. We note that interstellar reddening is insignificant because of the proximity of the $\operatorname{star}(d=122 \mathrm{pc})$ and its high galactic latitude $\left(b=-44^{\circ}\right)$; there is also no evidence for reddening in the observed Strömgren colours (Table 2).

We adopted standard relations for absolute magnitude as well as optical and infrared colours of main-sequence stars from the tables of Tokunaga (2000) and Drilling \& Landolt (2000). Given the observed $V$ magnitude and $\left(V-I_{\mathrm{C}}\right)$ colour of the system, we added the fluxes of hypothetical G0, K0 and K5 main-sequence companions. For the flux of the primary we used a suitable interpolation between $\mathrm{A} 7$ and $\mathrm{F} 0$ to reproduce the observed $V$ and $\left(V-I_{\mathrm{C}}\right)$. The infrared colours of those models were then compared to observations. The result, displayed in Fig. 14, shows the relative infrared excess of the observations and of some of the binary models compared to the expected infrared magnitudes.

Whereas the infrared $J$ and $H$ magnitude seem to indicate the presence of a companion, the $K$ magnitude argues against this idea; the $L$ magnitude is too uncertain to be useful. The infrared excess we calculated also depends on the uncertainties of the standard relations and the accuracy of our measurements. We conclude that we cannot pinpoint the nature of the companion of HD 209295 from the infrared data alone.

\section{FURTHER DISCUSSION OF THE OBSERVATI ONS}

\subsection{The secondary component and evolutionary history of the HD 209295 system}

We were unable to detect the secondary component of the HD 209295 system in the infrared. However, we can still obtain further constraints on its nature. The first constraint is the absence of ellipsoidal variability.

Morris (1985) derived theoretical expressions for system parameters of ellipsoidal variables from the observed light curves. Using his equation (6), a limb darkening coefficient of 0.59 from Claret (2000), a gravity darkening exponent of 0.84 (Claret 1999), and a conservative upper limit of $5 \mathrm{mmag}$ on the peak-to-peak amplitude of possible ellipsoidal variations of HD 209295, we can determine the minimum orbital inclination of the system as a function of secondary mass. The result is shown in Fig. 15.

Combining this information with the dependence of secondary mass on inclination from the mass function of the binary (upper panel of Fig. 8), we can derive a refined lower limit of the secondary mass. The result is $M_{2}>1.04 \mathrm{M}_{\odot}$. This lower limit on the secondary mass has two implications. First, it means that the primary is quite well within the Roche limit. Secondly, the secondary mass is very close to the upper limit $1.05 \mathrm{M}_{\odot}$ derived in 


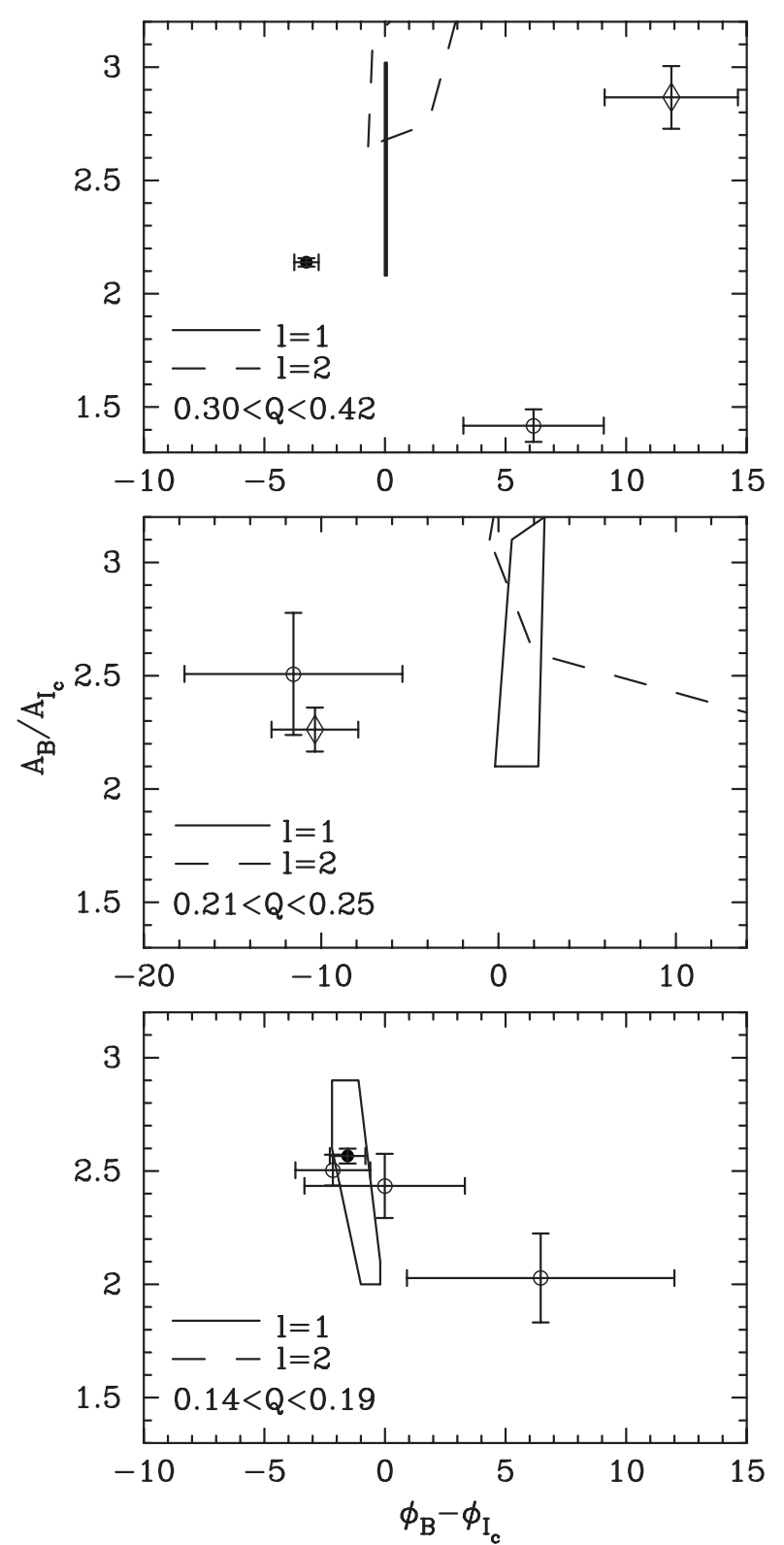

Figure 13. Comparison of theoretical and observed $B / I_{C}$ colour amplitude ratios and phase differences with observations. Upper panel: variations with frequencies less than 1.2 cycle $^{-1}$. Middle panel: variations with frequen-

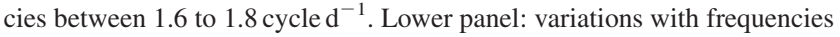
greater than 2.2 cycle $^{-1}$. The areas outlined by the full lines are theoretical predictions for $\ell=1$, and areas represented by dashed lines correspond to $\ell=2$. Symbols with error bars are the observed values. Filled circles correspond to independent modes, open circles to harmonics of the orbital period and diamonds to combination frequencies. Note the different abscissa scale in the middle panel. Regions of interest were also calculated for $\ell=3$, but they are off scale towards higher amplitude ratios. The $\ell=2$ area for $0.14<Q<0.19$ is off scale towards larger positive phase shifts.

Section 3.2.1, assuming a main-sequence secondary. In addition, the orbit of the system is also quite eccentric, which is surprising as one would have expected circularization to have taken place.

Claret, Giménez \& Cunha (1995) and Claret \& Cunha (1997) compared theoretical orbital circularization and synchronization times for main-sequence binaries with observations. From their results, and using the surface gravity of HD 209295 as an age

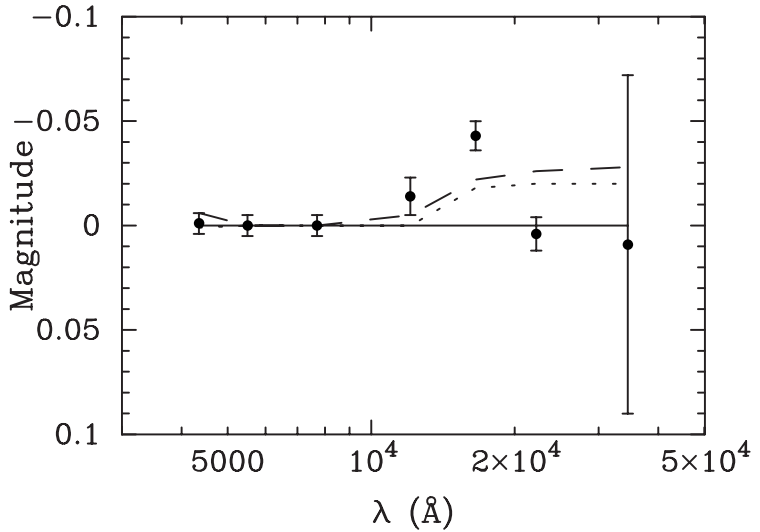

Figure 14. A search for infrared excess in the energy distribution of HD 209295. The solid horizontal line represents an interpolation of standard relations for A-type stars to the observed $\left(V-I_{\mathrm{C}}\right)$ colour of HD 209295. The dotted line is the same with a G0 main-sequence companion added, and the dashed line shows the expected colours if a K5 main-sequence companion were present. A K0 companion would be practically indistinguishable from a G0 star in this diagram. The filled circles with the error bars are the actual observations.

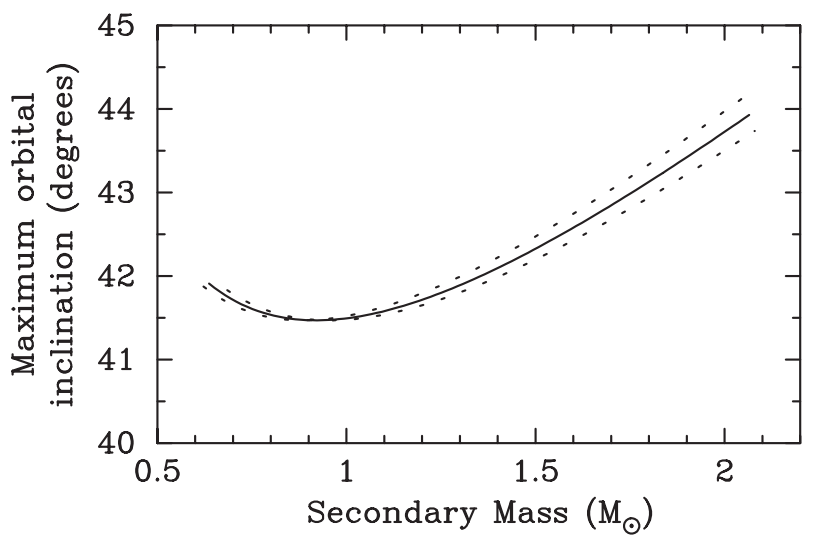

Figure 15. The maximum inclination of the binary orbit of HD 209295, consistent with the absence of ellipsoidal variability, as a function of secondary mass. The full line corresponds to a primary mass of $1.84 \mathrm{M}_{\odot}$, whereas the dotted lines delineate our $1 \sigma$ error estimate of $\pm 0.07 \mathrm{M}_{\odot}$.

indicator, we conclude that the orbital eccentricity of HD 209295 is inconsistent with normal main-sequence binary evolution. For a star similar in age to HD $209295(\log t \approx 8.86$ as inferred from comparison with pulsational and evolutionary models), one would expect $e<0.1$. There is reason to believe, therefore, that the secondary star may be a degenerate object. A white dwarf may have a mass in excess of $1.04 \mathrm{M}_{\odot}$. However, in such a scenario orbital circularization would again have taken place, because such a system must have undergone a previous mass transfer phase.

We investigated the ultraviolet (UV) fluxes of the object as measured by the TD-1 satellite (Thompson et al. 1978) and compared it to the optical uby fluxes. For this purpose, we converted the measured uby magnitude of HD 209295 to fluxes using the formulae of Gray (1998). We omitted the $v$ band because it is dominated by the $H_{\delta}$ line. A Kurucz model atmosphere with $T_{\text {eff }}=7750 \mathrm{~K}$ (cf. Section 1.1) and $\log g=4.3$ gave the best match. We then compared the predicted UV fluxes from this fit with the $T D-1$ measurements (Fig. 16). There is quite a strong UV excess in the $T D-1$ measurements at 1965 and $2365 \AA$ compared to 


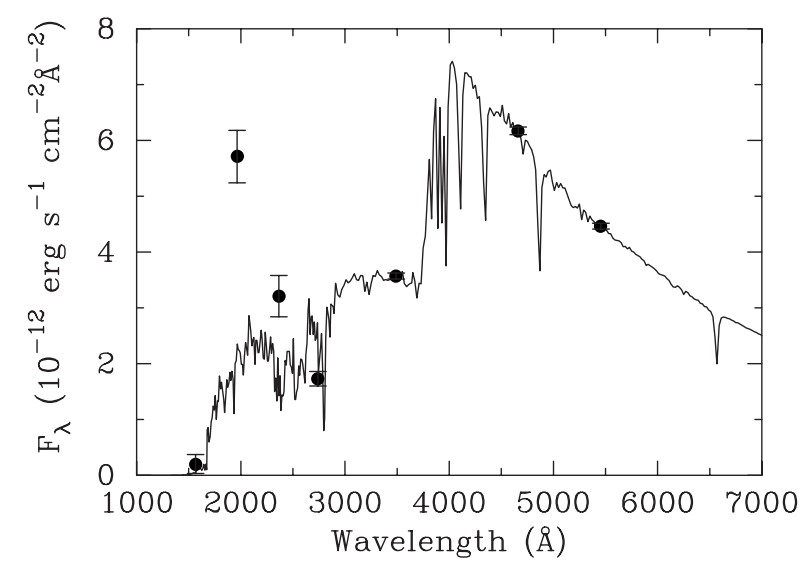

Figure 16. Comparison of optical uby and UV fluxes of HD 209295 to a $T_{\text {eff }}=7750 \mathrm{~K}, \log g=4.3$ Kurucz model atmosphere. A clear UV excess is notable.

the model atmosphere, but little flux at $1565 \AA$. The shape of this excess resembles an energy distribution of an object of $T_{\text {eff }} \approx 15000 \mathrm{~K}$. This is a suggestion that a white dwarf companion could be responsible.

Consequently, we attempted to fit some white dwarf star model atmospheres (Koester, private communication) for $\log g=8$ and $10000<T_{\text {eff }}<16000 \mathrm{~K}$ to this excess. The $V$ flux was normalized to the value predicted for a star at the distance of HD 209295. While we could reproduce the approximate shape of the UV excess, the resulting white dwarf luminosity is far too small. A white dwarf star would only be able to generate $0.1-0.2$ per cent of the observed UV excess. Hotter white dwarf models would be able to explain a larger fraction of the excess, but they would be inconsistent with the low TD- 1 flux at $1565 \AA$.

It is hard to imagine an astronomical object or a physical process which could be made responsible for this UV excess and still be consistent with other observations. A less-massive white dwarf is inconsistent with our mass constraints. A subdwarf would be seen in the optical and is inconsistent with the primary being a Population I star. A late-type active companion would be detected in X-rays. We need to leave the reason for the UV excess unanswered; it may be possible that the $T D-1$ measurements are in error. Unfortunately, the star was not observed by IUE (NASAESA 1999).

We also need to consider that the secondary may be a neutron star. In this case, the high orbital eccentricity would be a result of the supernova outburst, and the orbit would not be expected to circularize during the main sequence life span of the present primary (see Zahn 1977, whose work also suggests that the rotation of the primary should not be synchronized with the orbital motion of the compact secondary).

Comparing the orbital eccentricity and separation of the HD 209295 system with the X-ray binary population simulations of Terman, Taam \& Savage (1996) shows that the observed orbital parameters can be comfortably explained in this scenario. The undetected companion of HD 209295 could therefore be a neutron star, and the system will evolve into an intermediate-mass X-ray binary after the present primary has left the main sequence. The neutron star hypothesis is testable. The previous evolutionary phases should have left their marks in the chemical composition of HD 209295. However, the Strömgren metallicity index $\delta m_{1}=$ 0.016 and our spectra appear normal.

If the unknown companion of HD 209295 were a neutron star, one would also expect that it would be detectable in X-rays. However, the star has not been detected by ROSAT (Voges et al. 1999, 2000) or Einstein (Moran et al. 1996). Finally, the neutron star should have suffered an impulse from the supernova explosion, which might modify the space motion of the binary system. We therefore calculated the galactic $U, V$, and $W$ velocities of HD 209295 from its $\gamma$ velocity from Table 7, its Hipparcos parallax (see Section 1.1) and proper motion $\left[\mu_{\alpha} \cos \delta=26.84 \pm 0.59\right.$ mas, $\mu_{\delta}=-58.82 \pm 0.41$ mas as measured by the Hipparcos satellite (ESA 1997)]. We find $U=-21 \mathrm{~km} \mathrm{~s}^{-1}, V=-12 \mathrm{~km} \mathrm{~s}^{-1}$ and $W=+28 \mathrm{~km} \mathrm{~s}^{-1}$ (already corrected for the solar motion) which is not unusual for an early-F main-sequence star (cf. Gilmore \& Zeilik 2000). The neutron star interpretation for the secondary in the HD 209295 system therefore also has its weaknesses; we cannot at present identify the nature of this star with certainty.

\subsection{Evidence for forced oscillations}

In Section 3.4 we reported the discovery that many of the photometric frequencies are exact integer multiples of the orbital frequency. This raises the suspicion that they might not be free oscillation modes of the star, but are rather triggered by tidal interactions. Tidally induced non-radial oscillations have been searched for observationally, but the results have not been very convincing, with the possible exception of the slowly pulsating B star HD 177863 (De Cat 2001, Willems \& Aerts 2001). As there are quite a number of frequencies which are exact integer multiples of the orbital frequency, there is reason to believe that HD 209295 is the best case for forced oscillations. Further support for this interpretation needs to be sought. Some general information about tidal excitation is therefore useful.

This effect has been studied by several authors theoretically, most often in connection with neutron star/main-sequence binaries. For instance, Kumar, Ao \& Quatert (1995) showed that stellar p, f and low-order g-modes are not easily excitable through tidal effects, but that intermediate-order g-modes may be excited. These are, of course, exactly the modes in which $\gamma$ Dor stars (and SPB stars) pulsate.

Furthermore, tidally excited modes will have a shape adjusted to the gravitational potential of the exciting star. The tidal deformation can be decomposed into a linear superposition of the g- and p-mode oscillations of the star (Press \& Teukolsky 1977). Their dominating components are the $\ell=2,|m|=2$ modes (e.g. see Kosovichev \& Severnyj 1983). Unfortunately, we did not succeed in providing mode identifications for one or more of the variations suspected to be forced oscillations. However, if we are really dealing with forced oscillations, they should be approximately in phase at periastron, where the tidal force is at maximum.

We have already listed pulsational phases of the different modes at a periastron passage in Table 9. The phase values are similar and seem to show some alignment of the suspected tides. This is also the case for $f_{8}=5 f_{\text {orb}}$, assuming it is an $\ell=2,|m|=2$ mode, as there is a $180^{\circ}$ phase ambiguity. The variations are, however, not perfectly in phase, possibly as a result of non-adiabatic effects.

\subsection{The photometric and radial velocity amplitudes}

It is somewhat puzzling that there is little correspondence in amplitude between the photometric and radial velocity data. The two highest-amplitude $\gamma$ Dor modes in the photometry are undetected in the radial velocities. While the range in frequency of $\delta$ Sct instability is in approximate agreement in the photometry and 
Table 10. A comparison of simultaneous $V$-filter light and radial velocity amplitudes for $\gamma$ Dor stars from the literature.

\begin{tabular}{lccccc}
\hline Star & $\begin{array}{c}2 K \\
\left(\mathrm{~km} \mathrm{~s}^{-1}\right)\end{array}$ & $\begin{array}{c}\Delta V \\
(\mathrm{mmag})\end{array}$ & $\begin{array}{c}2 K / \Delta V \\
\left(\mathrm{~km} \mathrm{~s}^{-1} \mathrm{mag}^{-1}\right)\end{array}$ & $\ell$ & Ref. \\
\hline$\gamma$ Dor & $0.6 \pm 0.2$ & $23 \pm 1$ & $26 \pm 9$ & 3 & 1 \\
& $2.6 \pm 0.2$ & $27 \pm 1$ & $96 \pm 8$ & 1 & \\
9 Aur & $1.2 \pm 0.2$ & $13 \pm 1$ & $92 \pm 8$ & 1 & \\
& $1.7 \pm 1.2$ & $35 \pm 1$ & $48 \pm 34$ & 3 & 2,3 \\
HR 8330 & $3.0 \pm 0.8$ & $20 \pm 1$ & $148 \pm 39$ & 3 & 2,3 \\
HD 68192 & $3.1 \pm 1.1$ & $18 \pm 1$ & $172 \pm 61$ & & 2,3 \\
HD 209295 & $2.2 \pm 0.1$ & $15 \pm 1$ & $350 \pm 20$ & 2 & 4,5 \\
& $<1$ & $38.9 \pm 0.2$ & $101 \pm 10$ & & 6 \\
& $<1$ & $28.7 \pm 0.2$ & $<26$ & 1 & 7 \\
& & $<35$ & 1 & 7 \\
\hline
\end{tabular}

References: 1: Balona et al. (1996); 2: Zerbi et al. (1997); 3: Aerts \& Krisciunas (1996); 4: Kaye et al. (1999b); 5: Aerts \& Kaye (2001); 6: Kaye et al. (1999c); 7: this paper.

radial velocities, there is no clear match to individual frequencies between the two data sets.

Breger, Hutchins \& Kuhi (1976) determined the ratios of velocity to light amplitudes in several $\delta$ Sct stars and found values between $2 K / \Delta V$ of $50-125 \mathrm{~km} \mathrm{~s}^{-1} \mathrm{mag}^{-1}$. Using this value for the $\delta$ Sct pulsations in HD 209295, we would expect the photometrically detected modes to have radial velocity amplitudes smaller than $0.2 \mathrm{~km} \mathrm{~s}^{-1}$, and hence they would not be detected. However, we detected radial velocity variations with amplitudes of around $2 \mathrm{~km} \mathrm{~s}^{-1}$ in the $\delta$ Sct range, which should then generate photometric $V$ amplitudes of at least $15 \mathrm{mmag}$, but there is no trace of them. The only plausible explanation is that the short-period variations in the spectroscopy are not due to pulsation.

We can get limits to the radial velocity amplitude of the two independent $\gamma$ Dor modes of HD 209295 by fitting the radial velocities with the photometric periods. This results in an upper limit of $1 \mathrm{~km} \mathrm{~s}^{-1}$ for the amplitude of both modes. We then obtain $2 K / \Delta V<26 \mathrm{~km} \mathrm{~s}^{-1} \mathrm{mag}^{-1}$ for $f_{1}$ and $2 K / \Delta V<35 \mathrm{~km} \mathrm{~s}^{-1} \mathrm{mag}^{-1}$ for $f_{2}$.

In Table 10 we show all available amplitude determinations from photometric and radial velocity measurements of $\gamma$ Dor stars from the literature. We only considered data sets in which simultaneous radial velocity and photometry was obtained to avoid being sensitive to amplitude variations. Only those cases where individual mode periods could be resolved are listed.

It appears that not only is there an order of magnitude spread in the $2 K / \Delta V$ values for the different stars, but also for different modes of the same star. The limits we obtained for HD 209295 are at the lower end of the range to be found in Table 1. However, the lowest $2 K / \Delta V$ values were obtained for modes of $\ell=3$, not for $\ell=1$ as suggested for the two independent modes of HD 209295.

Aerts \& Krisciunas (1996) offered an explanation for such diverse behaviour: the photometric variations are mostly due to temperature variations, which hardly affect the radial velocities. In addition Aerts \& Krisciunas (1996) suggest that, due to stellar rotation, toroidal corrections become important. These may be the cause for the large scatter in $2 K / \Delta V$ in Table 10. High-resolution, high signal-to-noise ratio spectra of the more rapidly rotating $\gamma$ Dor stars with simultaneous photometry are necessary to test this hypothesis.

\section{THEORY}

We have carried out a stability analysis of the pulsations to check
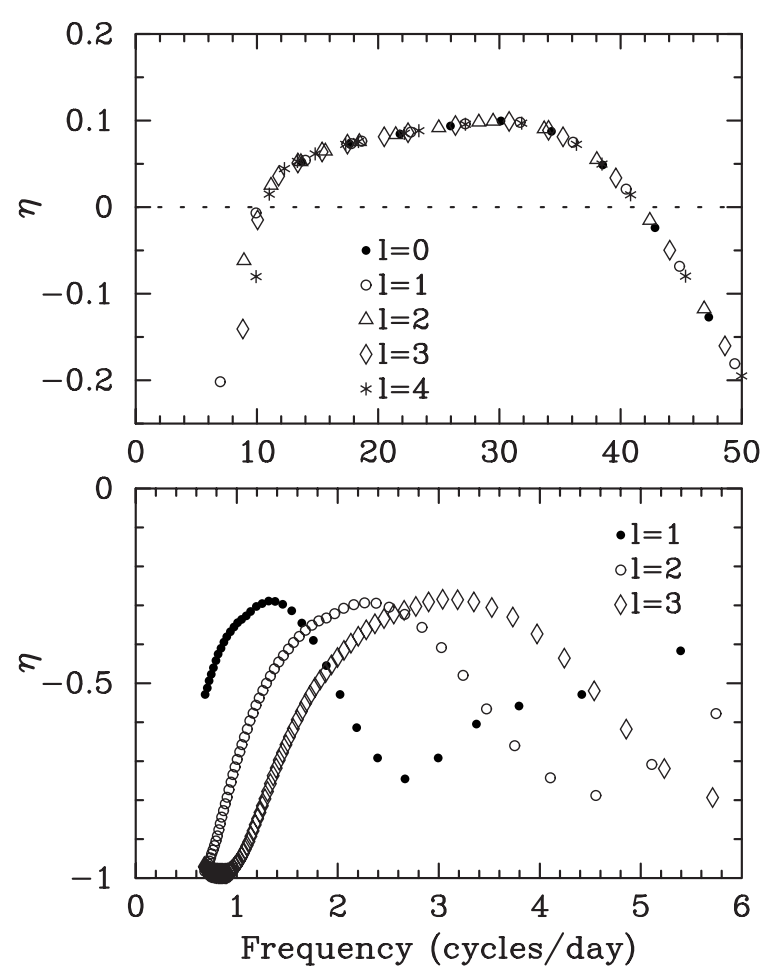

Figure 17. Normalized linear growth rates, $\eta$, for a model matching the effective temperature and surface gravity HD 209295. $\eta=1$ means that a mode is driven throughout the whole model, whereas $\eta=-1$ means that a mode is damped throughout the whole model; modes with $\eta>0$ are pulsationally unstable. Upper panel: the whole computed frequency range. Lower panel: the $\gamma$ Dor pulsation frequency domain. In certain frequency regions (depending on $\ell$ ), less damping is present.

whether the inferred evolutionary state of HD 209295 is consistent with its pulsational behaviour, and whether tidally induced oscillations are reasonable.

\subsection{Stability analysis}

We used the Warsaw-New Jersey stellar evolution and the NADROT pulsation codes to generate a series of models for HD 209295. We then investigated the pulsation modes for stability. The results for the model which best matches the parameters of HD 209295 $\left(M=1.8 \mathrm{M}_{\odot}, \log T_{\text {eff }}=3.88, \log L=1.10, \log g=4.03\right)$ are shown in Fig. 17.

The observed frequency domain of the $\delta$ Sct pulsations of HD 209295 is very well reproduced. The unstable frequency range does not depend on $\ell$, which is also consistent with the observations. $\gamma$ Doradus modes are not driven in our models, as this currently requires a special treatment of convection (Guzik et al. 2000). In our computations we used the standard mixinglength convection theory and we ignored the Lagrangian perturbation of the convective energy flux when computing oscillations. Our attempts to reproduce the results by Guzik et al. (2000) concerning pulsational instability were unsuccessful, and we plan to study this matter in the future.

It is interesting to note that there are frequency regions in which damping is not as strong, and that they depend on $\ell$. We performed these computations up to $\ell=8$, and found that the trend is maintained. The $\ell=1$ and $\ell=2$ frequency regions coincide with the ones actually observed. 


\subsection{Tidal excitation}

We investigated the possibility of tidally excited oscillations in HD 209295 by determining the amplitude of tidally induced radialvelocity variations using the expressions derived by Willems \& Aerts (2001). We calculated both the free and the forced oscillations of a $1.8 \mathrm{M}_{\odot}$ stellar model in the linear, adiabatic approximation. Because the amplitude may become quite large in the adiabatic approximation, we restricted ourselves to calculating the radial-velocity variations at orbital periods for which the relative differences between the forcing frequencies of the dynamic tides and the eigenfrequencies of the free oscillation modes are not too small (for details see Willems \& Aerts 2001).

The resulting amplitudes of the tidally induced radial-velocity variations seen by an observer are displayed in Fig. 18 as a function of the orbital period. The companion mass is assumed to be $1.5 \mathrm{M}_{\odot}$, which corresponds to an orbital inclination of $31.2^{\circ}$ and to a rotational frequency between $f_{\text {rot }}=1.635 \mathrm{~d}^{-1}$ and $f_{\text {rot }}=1.998 \mathrm{~d}^{-1}$.

A high peak in the amplitudes is seen to occur near the observed orbital period of HD 209295. The peak results from the resonant excitation of the modes $g_{14}^{+}$and $g_{16}^{+}$by the dynamic tides associated with the forcing frequencies $3 f_{\text {orb }}$ and $4 f_{\text {orb }}$, respectively. Both tides are of spherical degree $\ell=2$ and azimuthal order $m=-2$. This result is quite encouraging, as a signal at $3 f_{\text {orb }}$ was detected in the photometry, and one at $4 f_{\text {orb }}$ was suspected. On the other hand, not all of the detected orbital harmonics are excited in the model, but they can be reconciled with linear combinations of these two signals.

We need to make several remarks here. First, the above 'solution' is probably not unique. In addition, the calculations have been performed using an adiabatic code, so they show the presence of the resonances, but not the real amplitudes. Secondly, attempts with lower companion masses (and thus lower rotational

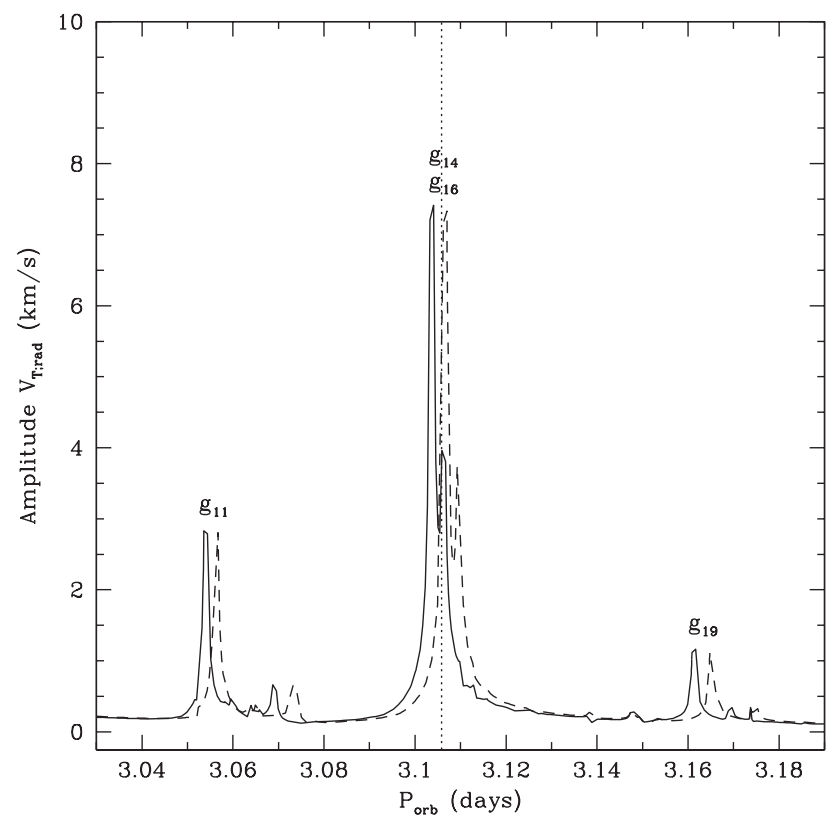

Figure 18. Observed amplitude of the tidally induced radial velocity variations in a model for HD 209295. The full line corresponds to a rotational frequency $f_{\text {rot }}=1.852 \mathrm{~d}^{-1}$ and the dashed line to a rotational frequency $f_{\text {rot }}=1.854 \mathrm{~d}^{-1}$. The orbital period of HD 209295 is indicated by the dotted vertical line. frequencies) were not very successful. This is interesting, as it may help to constrain the nature of the orbital companion of HD 209295.

We conclude that resonant excitation is possible, but for precise results there are still too many degrees of freedom. The prospect of developing tides as a probing tool is a promise for future studies.

\section{CONCLUSIONS AND OUTLOOK}

By means of $128 \mathrm{~h}$ of time-resolved high-resolution spectroscopic and $280 \mathrm{~h}$ of time-series photometric observations, we have shown that HD 209295 is not only the first pulsating star to be a member of two classes (a $\gamma$ Dor as well as a $\delta$ Sct star), but we have also discovered good evidence for the presence of tidally excited pulsation modes. The binary companion is probably a neutron star or a white dwarf.

These findings make HD 209295 a rather interesting astrophysical laboratory. We were able to confront photometric and spectroscopic mode identification methods. Whereas the method by Telting \& Schrijvers (1997) yielded reasonable assignments for the two independent modes dominating the photometry (both are $\ell=1,|m|=1$ ), analysing the photometric colour amplitude ratios and phase differences did not yield any meaningful results. The necessity of using as many mode identification methods as possible to secure reliable results is stressed.

We showed that the presence of tidally induced modes can be explained by theoretical models, but not all the observations can be understood. It would be important to calculate more realistic models of tidal interaction.

We do not fully understand the wealth of information provided by the variations in HD 209295. Large coordinated efforts will be required for further progress as our data are already fairly extensive. Another multisite photometric and spectroscopic campaign on an even larger scale would be desirable. This should be carried over a long time-base in order to resolve the $\gamma$ Dor pulsations, and a large data set is needed to detect more $\delta$ Sct modes. Large telescopes are required for the spectroscopy to obtain high time-resolution and a high signal-to-noise ratio. This is necessary for identifying further modes and for detailed lineprofile analysis. Photometric observations in more filters than previously obtained are necessary, perhaps extending the wavelength range into the near infrared. UV and infrared spectroscopy would be useful in a search for the companion star, and an abundance analysis may reveal an unusual chemical composition caused by the possible previous evolution of the system through mass transfer, common envelope and/or supernova stages.

\section{ACKNOWLEDGMENTS}

We are indebted to Lisa Crause for obtaining and reducing the infrared photometry of HD 209295 at our request and for commenting on a draft version of this paper. DJJ and BW thank the British Particle Physics and Astronomy Research Council (PPARC) for post-doctoral research fellowships. DJJ would also like to acknowledge the continued positive influences of Mrs J Pryer, for much love and support during this work, and the Royal Society for a European research grant. ERC thanks S. Potter, D. Romero and E. Colmenero for their support. AAP would like to thank L. A. Balona and the staff of the SAAO (Cape Town) for their hospitality during his visit to the institute. BW is grateful to 
Antonio Claret for providing a $1.8-\mathrm{M}_{\odot}$ stellar model. GH thanks Darragh O'Donoghue for giving him one night of his observing time for this project and Detlev Koester for providing white dwarf model atmospheres. GH also wishes to express his gratitude to several colleagues for helpful comments: Brian Warner, Marten van Kerkwijk, Matt Burleigh and Stephen Potter. Ennio Poretti and Kevin Krisciunas are thanked for commenting on a draft version of this paper.

\section{RE F E R E N CES}

Aerts C., Kaye A. B., 2001, ApJ, 553, 814

Aerts C., Krisciunas K., 1996, MNRAS, 278, 877

Balona L. A., 2000, MNRAS, 318, 289

Balona L. A., Evers E. A., 1999, MNRAS, 302, 349

Balona L. A. et al., 1996, MNRAS, 281, 1315

Baranne A. et al., 1996, A\&AS, 119, 373

Barker E. S., Evans D. S., Laing J. D., 1967, Bull. Roy. Obs., No. 130

Bedding T. R. et al., 2002, in Aerts C., Bedding T. R., ChristensenDalsgaard J., eds, ASP Conf. Ser. Vol. 259, Radial and Nonradial Oscillations as Probes of Stellar Physics. Astron. Soc. Pac., San Fransisco, in press

Breger M., 1975, in Sherwood V., Plaut L., eds, Proc. IAU Symp. 67, Variable Stars and Stellar Evolution. Reidel, Dordrecht, p. 231

Breger M., Montgomery M. H., 2000, in Breger M., Montgomery M. H., eds, ASP Conf. Ser. Vol. 210, Delta Scuti and Related Stars. Astron. Soc. Pac, San Francisco

Breger M., Hutchins J., Kuhi L. V., 1976, ApJ, 210, 163

Breger M. et al., 1993, A\&A, 271, 482

Carter B. S., 1990, MNRAS, 242, 1

Claret A., 1999, A\&AS, 131, 395

Claret A., 2000, A\&A, 363, 1081

Claret A., Cunha N. C. S., 1997, A\&A, 318, 187

Claret A., Giménez A., Cunha N. C. S., 1995, A\&A, 299, 724

Crawford D. L., 1979, AJ, 84, 1858

Cunha M. S., Gough D. O., 2000, MNRAS, 319, 1020

De Cat P., 2001, PhD thesis, Katholieke Universiteit Leuven

Drilling J. S., Landolt A. U., 2000, in Cox A. N., ed., Allen's Astrophysical Quantities, 4th edn. Springer Verlag, Berlin, p. 392

Eggleton P., 1983, ApJ, 268, 368

ESA, 1997, The Hipparcos and Tycho catalogues, ESA SP-1200. ESA Publications Division, Noordwijk

Eyer L., Grenon M., 1998, in Deubner F. L., Christensen-Dalsgaard J., Kurtz D. W., eds, New Eyes to See Inside the Sun and Stars, p. 291

Garrido R., 2000, in Breger M., Montgomery M., eds, ASP Conf. Ser. Vol. 210, Delta Scuti and Related Stars. Astron. Soc. Pac., San Francisco, p. 67

Gilmore G. F., Zeilik M., 2000, in Cox A. N., ed., Allen's Astrophysical Quantities, 4th edn. Springer Verlag, Berlin, p. 493

Glass I. S., 1973, MNRAS, 164, 155

Gough D. O., 2000, in Brekke P., Fleck B., Gurman J. B., eds, Proc. IAU Symp. 203, Recent Insights into the Physics of the Sun and Heliosphere. Astron. Soc. Pac., San Fransisco, p. 1

Gray R. O., 1998, AJ, 116, 482
Gray R. O., Corbally C. J., 1994, AJ, 107, 742

Grenier S., Burnage R., Faraggiana M., Gerbaldi M., Delmas F., Gómez A. E., Sabas V., Sharif L., 1999, A\&AS, 135, 503

Guzik J. A., Kaye A. B., Bradley P. A., Cox A. N., Neuforge C., 2000, ApJ, 542, L57

Handler G., 1999a, MNRAS, 309, L19

Handler G., 1999b, IBVS, 4817

Handler G., Shobbrook R. R., 2002, MNRAS, 333, 251 (Paper I, this issue)

Handler G. et al., 1997, MNRAS, 286, 303

Handler G. et al., 2000, MNRAS, 318, 511

Houk N., Cowley A. P., 1975, Michigan Spectral Catalogue, Vol. 1. Kluwer, Dordrecht

Kaye A. B., Handler G., Krisciunas K., Poretti E., Zerbi F. M., 1999a, PASP, 111,840

Kaye A. B., Henry G. W., Fekel F. C., Hall D. S., 1999b, MNRAS, 308, 1081

Kaye A. B. et al., 1999c, AJ, 118, 2997

Kjeldsen H., Bedding T. R., Viskum M., Frandsen S., 1995, AJ, 109, 1313

Koen C., Van Rooyen R., Van Wyk F., Marang F., 1999, MNRAS, 309, 1051

Kosovichev A. G., Severnyj A. B., 1983, Pis'ma Astron. Zh., 9, 424

Kumar P., Ao C. O., Quataert E. J., 1995, ApJ, 449, 294

Kurtz D. W., Martinez P., 2000, Baltic Astron., 9, 253

Kurucz R. L., 1991, Van Vleck Obs. Contr., 11, 27

Martinez P., Koen C., 1994, MNRAS, 267, 1039

Montgomery M. H., O’Donoghue D., 1999, Delta Scuti Star Newsletter 13. Univ. Vienna, Vienna, p. 28

Moran E. C., Helfand D. J., Becker R. H., White R. L., 1996, ApJ, 461, 127

Morris S. L., 1985, ApJ, 295, 143

NASA-ESA, 1999, Merged Log of IUE observations. Available online from http://vizier.u-strasbg.fr/cgi-bin/VizieR, catalogue No. VI/99

Pamyatnykh A. A., Dziembowski W. A., Handler G., Pikall H., 1998, A\&A, 333,141

Press W. H., Teukolsky S. A., 1977, ApJ, 213, 183

Rodriguez E., Breger M., 2001, A\&A, 366, 178

Rodriguez E., López-González M. J., López de Coca P., 2000, A\&AS, 144, 369

Shobbrook R. R., 2000, J. Astron. Data, 6, 4E

Sperl M., 1998, MSc thesis, Univ. Vienna

Telting J. H., Schrijvers C., 1997, A\&A, 317, 723

Terman J. L., Taam R. E., Savage C. O., 1996, MNRAS, 281, 552

Thompson G. I., Nandy K., Jamar C., Monfils A., Houziaux L., Carnochan D. J., Wilson R., 1978, Catalogue of stellar ultraviolet fluxes (TD1). The Science Research Council, UK

Tokunaga A. T., 2000, Cox A. N., ed., Allen's Astrophysical Quantities, 4th edn. Springer Verlag, Berlin, p. 151

Twarog B. A., 1980, ApJS, 44, 1

Voges W. et al., 1999, A\&A, 349, 389

Voges W. et al., 2000, IAU Circ., 7432

Watson R. D., 1988, Ap\&SS, 140, 255

Willems B., Aerts C., 2002, A\&A, 384, 441

Zahn J.-P., 1977, A\&A, 57, 383

Zerbi F. M. et al., 1997, MNRAS, 290, 401

This paper has been typeset from a $\mathrm{T}_{\mathrm{E}} \mathrm{X} / \mathrm{L} \mathrm{T} \mathrm{E} X$ file prepared by the author. 